Article

\title{
Noninferior Solution Grey Wolf Optimizer with an Independent Local Search Mechanism for Solving Economic Load Dispatch Problems
}

\author{
Jianzhong Xu ${ }^{1}$, Fu Yan ${ }^{1, *}$, Kumchol Yun ${ }^{1,2}$, Lifei Su ${ }^{3}$, Fengshu Li ${ }^{1}$ and Jun Guan ${ }^{4}(\mathbb{D}$ \\ 1 School of Economics and Management, Harbin Engineering University, 145 Nantong Street, \\ Harbin 150001, China; xujianzhongxjz@163.com (J.X.); kumchol-yun@hrbeu.edu.cn (K.Y.); \\ lifengshuhello@163.com (F.L.) \\ 2 Faculty of Mechanics, Kim Il Sung University, Pyongyang 950003, Democratic People's Republic of Korea \\ 3 College of Resources and Environment, Northeast Agricultural University, 600 Changjiang Road, \\ Harbin 150030, China; Lifei_SU@163.com \\ 4 College of Economics and Management, Northeast Forestry University, 26 Hexing Road, \\ Harbin 150040, China; guanjun_89@126.com \\ * Correspondence: yanfuphd@hrbeu.edu.cn; Tel.: +86-188-4516-2935
}

Received: 5 May 2019; Accepted: 10 June 2019; Published: 13 June 2019

\begin{abstract}
The economic load dispatch (ELD) problem is a complex optimization problem in power systems. The main task for this optimization problem is to minimize the total fuel cost of generators while also meeting the conditional constraints of valve-point loading effects, prohibited operating zones, and nonsmooth cost functions. In this paper, a novel grey wolf optimization (GWO), abbreviated as NGWO, is proposed to solve the ELD problem by introducing an independent local search strategy and a noninferior solution neighborhood independent local search technique to the original GWO algorithm to achieve the best problem solution. A local search strategy is added to the standard GWO algorithm in the NGWO, which is called GWOI, to search the local neighborhood of the global optimal point in depth and to guarantee a better candidate. In addition, a noninferior solution neighborhood independent local search method is introduced into the GWOI algorithm to find a better solution in the noninferior solution neighborhood and ensure the high probability of jumping out of the local optimum. The feasibility of the proposed NGWO method is verified on five different power systems, and it is compared with other selected methods in terms of the solution quality, convergence rate, and robustness. The compared experimental results indicate that the proposed NGWO method can efficiently solve ELD problems with higher-quality solutions.
\end{abstract}

Keywords: grey wolf optimizer (GWO); noninferior solution; local search mechanism; economic load dispatch problems (ELD); optimization algorithms

\section{Introduction}

Optimization problems widely exist in various fields in real-life. Some of these optimization problems are simple, while others are very complex due to nonconvex objective functions and complex model constraints. For complex optimization problems, a typical characteristic is the minimum or maximum objective function that is subject to heavy equality and/or inequality constraints. The economic load dispatch problem (ELD) is a famous complex power system operation optimization problem. ELD is a computational process, in which the total demanded generation is optimally allocated to each generation unit in operation by minimizing the selected cost criterion while also satisfying the total demand, transmission losses, and a set of physical and operational constraints imposed by the generators and system limitations $[1,2]$. The optimization study of the ELD problem is 
implied to be of great significance, because it can effectively save energy and provide a prioritization scheme for the control of real-time energy management power systems to guide power generation companies to implement sustainable development strategies. In addition, ELD is one of the important contents of power grid production and operation activities. Improving the economic production level of power grids can produce significant economic and social benefits. In general, the reasonable allocation of economic burden can save fuel by $0.5 \sim 2 \%$, the efficiency of the unit economic combination can reach $1 \sim 2.5 \%$, and the network loss correction benefit is $0.05 \sim 0.5 \%$. Therefore, it is very important to optimize the economic dispatching of power plants.

Classic mathematical optimization techniques have been employed in previous attempts to solve the ELD problem, such as the fast lambda iteration method [3], quadratic programming [4], the gradient method [5], the interior point technique [6], the linear programming algorithm [7], dynamic programming [8], and the Lagrange relaxation algorithm [9]. However, in these methods, when encountering nonconvex objective function with complex constraints and highly nonlinear, nonconvex, and noncontinuous features with many local optima, the practical constraints of the generating units and the network have to be simplified or ignored, owing to the limits of these methods $[10,11]$. Faced with the inability to solve complex ELD problems with traditional methods, researchers turned to metaheuristic and evolutionary optimization techniques, such as the genetic algorithm (GA) [12], particle swarm optimization (PSO) [13], the cuckoo search algorithm (CSA) [14], the artificial bee colony algorithm (ABC) [15], the chaotic bat algorithm (CBA) [16], harmony search (HS) [17], grey wolf optimization (GWO) [18], hybrid grey wolf optimization (HGWO) [19], the differential evolution PSO (DE-PSO) method [20], the harmony search DE (HS-DE) method [21], and improved PSO (IPSO) [22], and achieved good expected results as these techniques could handle various complex operating constraints, such as prohibited operating zones (POZ) and generators' ramp-up and ramp-down [11]. The heuristic algorithms that are listed above for solving complex ELD problems can be summarized into three categories [11]: (I) the techniques applied to ELD problems in their original versions; (II) the modified versions of the first category; and, (III) the hybrid methods of the two original versions of the first category. However, except for the GWO algorithm, all of the meta-heuristic or evolutionary optimization techniques that are mentioned above require the algorithm parameters to be artificially set or adjusted to obtain better optimization performance, and once the algorithm-related parameter settings are unreasonable, it is difficult to obtain the desired results. Therefore, it is more advantageous to choose the GWO algorithm to optimize the ELD problem, since good optimization results can be obtained without adjusting any algorithm parameters by GWO algorithm. Moreover, its algorithm principle and structure are very simple and easy to program.

Although metaheuristic and evolutionary algorithms have made considerable progress in solving complex ELD problems when compared with traditional mathematics-based methods, they still face considerable challenges in solving highly nonconvex ELD problems. The no free lunch (NFL) theorem can scientifically explain this phenomenon. According to NFL, it is difficult to find a meta-heuristic that is best suited for solving all optimization problems [23]. In other words, one approach may show very promising results on a particular class of problems, but the same algorithm may show poor results on a different set of problems [24]. Therefore, more researchers improve the current approaches or propose new meta-heuristics for solving different complex problems every year, such as the dragonfly algorithm, is hybridized with the improved Nelder-Mead algorithm (INMDA) for function optimization and multilayer perceptron training [25], the dynamically dimensioned search is improved by embedding with piecewise opposition-based learning (DDS-POBL) for global optimization [26], and this also motivates our attempts in this paper to improve the GWO algorithm for solving complex ELD problems.

The GWO algorithm is a recently proposed, yet advanced, meta-heuristic technique that was inspired by the hierarchy of grey wolf populations and Mirjalilili et al. developed it in 2014 [24]. In GWO, according to the different role of the grey wolf in advancing the hunting process, pack members are divided into four different groups: alpha, beta, delta, and omega. When compared with other algorithms, the GWO has a simpler algorithm structure, and no algorithm parameters need to be 
adjusted, except for setting the population size and the maximum number of iterations. In addition, two powerful operations parameters are designed to maintain the exploration and exploitation to avoid local optima stagnation [27]. These remarkable advantages make GWO a widely studied and applied technique in practical optimization problems, such as feature selection [28], training multilayer perceptron (MLP) networks [29], optimizing support vector machines [30], clustering applications [31], design and tuning controllers [32], ELD problems [18,19,33], path planning [34], and welding production scheduling [35]. However, as a newly proposed algorithm, the GWO algorithm still has the drawback of the NFL theorem, which states that no optimization algorithm is suitable for all optimization problems. The population diversity may decrease with an increase in the number of iterations, easily falling into local optimal solutions, since all three alpha, beta, and gamma wolves are likely to converge to the same point (solution).

In the standard GWO algorithm, the first three best global optimal solutions (alpha, beta, and gamma wolves) accelerate the convergence rate of the algorithm, but the neglect of the local optimal solution in each iteration weakens the search diversity of the GWO. In addition, the standard GWO has no local search capability for noninferior solution domains, so it easily becomes stuck at a local minimum point, and thus cannot effectively search for other possible global optimal regions. Therefore, an improved novel GWO algorithm (NGWO) that is based on a local optimal search and an independent local search for noninferior solution domains is proposed. In the NGWO algorithm, the first three best wolves in the current iteration are used to replace the first three best wolves that were obtained so far by the standard GWO algorithm for searching the population. In the iterative processes, when the error between the optimal fitness found by some particles and the current optimal fitness of the population is very small, the solution that was found by this particle is a noninferior solution, and there may be a better solution in its field. These individuals no longer move toward the global optimal solution but search for a better solution in their own neighborhood. Therefore, the search mechanism of the NGWO is useful for enhancing the search ability and increasing the chance of the algorithm jumping out of a local optimal solution.

The contributions of this paper are listed, as follows:

1. The first three local optimal solutions of the current iteration are used to replace the alpha, beta, and delta of the standard GWO algorithm for searching the population.

2. A local independent search mechanism for noninferior solutions is introduced in the standard GWO algorithm to avoid local optimization and to find more promising solutions.

3. The NGWO algorithm is proposed based on 1 and 2 and is applied to solve complex ELD problems.

The rest of this paper is structured, as follows: Section 2 presents the proposed NGWO algorithm, Section 3 provides the formulation of the ELD problem, Section 4 addresses the methodology of NGWO for solving ELD problems, and Section 5 presents the conclusions and future work.

\section{The Proposed NGWO Algorithm}

It is necessary to investigate the relative efficiency of each improved constituent when solving the ELD problem since the proposed NGWO algorithm is an improved version of the standard GWO algorithm, and thus four different algorithm versions are investigated:

- The basic GWO algorithm: The standard GWO algorithm is chosen as a comparison algorithm to compare the performance in solving different ELD cases with the other three improved versions.

- The compared GWOI algorithm: The standard GWO algorithm is improved by changing the strategy of searching the population, but without considering the case of a noninferior solution.

- The compared GWOII algorithm: The standard GWO algorithm is improved by only introducing the local independent search mechanism for the noninferior solution.

- The proposed NGWO algorithm: The standard GWO algorithm integrated with both the GWOI and GWOII methods. 


\subsection{The Basic GWO Algorithm}

GWO is a metaheuristic technique. The inspiration for the basic GWO algorithm is the hunting mechanism and social leadership hierarchy of grey wolves in nature. Grey wolves are considered as the top predators among animals and they have a strict leadership hierarchy. This leadership hierarchy generally consists of four different levels of groups: alpha $(\alpha)$, beta $(\beta)$, delta $(\delta)$, and omega $(\omega)$, where $\alpha, \beta$, and $\delta$ represent the first three best wolves (solutions) and the rest of the wolves (candidate solutions) are $\omega$. In the predation process, the prey is driven to the predation area by encircling under the guidance of the first three optimal grey wolves $(\alpha, \beta$, and $\delta)$. The encircle mechanism can be described by mathematical equations as [24]:

$$
\begin{gathered}
\vec{D}=\left|\vec{C} \cdot \vec{X}_{p}(t)-\vec{X}(t)\right| \\
\vec{X}(t+1)=\vec{X}_{p}(t)-\vec{A} \cdot \vec{D}
\end{gathered}
$$

where $t$ is the current iteration, $\vec{C}=2 \cdot \vec{r}_{2}$ and $\vec{A}=2 \cdot \vec{a} \cdot \vec{r}_{1}-\vec{a}$ indicates random vectors and they are used for balancing the exploration and exploitation, $\vec{X}_{p}$ represents the position vector of the prey, $\vec{X}$ is the position vector of a grey wolf, $\vec{a}$ is a control parameter that linearly decreases from 2 to 0 , and $\vec{r}$ and $\vec{r}_{2}$ are the random vectors over the range 0 and 1 .

In the basic GWO algorithm, the first three best wolves $(\alpha, \beta$, and $\delta)$ are considered to have better knowledge regarding the potential location of prey and they are responsible for guiding $\omega$ to hunt prey, so the other wolves $(\omega)$ can update their positions according to $\alpha, \beta$, and $\delta$. The following mathematical models are modeled in this regard [23]:

$$
\begin{aligned}
& \vec{D}_{\alpha}=\left|\vec{C}_{1} \cdot \vec{X}_{\alpha}-\vec{X}\right| \\
& \vec{D}_{\beta}=\left|\vec{C}_{2} \cdot \vec{X}_{\beta}-\vec{X}\right| \\
& \vec{D}_{\delta}=\left|\vec{C}_{3} \cdot \vec{X}_{\delta}-\vec{X}\right| \\
& \vec{X}_{1}=\vec{X}_{\alpha}-\vec{A}_{1} \cdot \vec{D}_{\alpha} \\
& \vec{X}_{2}=\vec{X}_{\beta}-\vec{A}_{2} \cdot \vec{D}_{\beta} \\
& \vec{X}_{3}=\vec{X}_{\delta}-\vec{A}_{3} \cdot \vec{D}_{\delta} \\
& \vec{X}(t+1)=\frac{\vec{X}_{1}+\vec{X}_{2}+\vec{X}_{3}}{3}
\end{aligned}
$$

where $\vec{X}_{\alpha}, \vec{X}_{\beta}$, and $\vec{X}_{\delta}$ are the positions of the alpha $(\alpha)$, beta $(\beta)$, and delta $(\delta)$, respectively; $\vec{D}_{\alpha}, \vec{D}_{\beta}$, and $\vec{D}_{\delta}$ indicate the encircle step sizes of $\alpha, \beta$, and $\delta$, respectively; $\vec{C}_{1}, \vec{C}_{2}$, and $\vec{C}_{3}$ and $\vec{A}_{1}, \vec{A}_{2}$, and $\vec{A}_{3}$ are the random vectors; $\vec{X}$ is the position vector of the current individual, and $t$ represents the number of current iterations.

\subsection{The Compared GWOI Algorithm}

From the basic GWO algorithm, it can be seen that this algorithm only considers the global search in the search mechanism and lacks the local search for the population. According to Res. [36], the global search is a rough search in the whole search space and the local search is a deep search in the neighborhood of the current optimal solution. If the algorithm only adopts a single global search method, once the particles guiding the global search fall into the local optimal situation (as shown in Figure 1), the algorithm search will easily stop near the local optimal solution. 


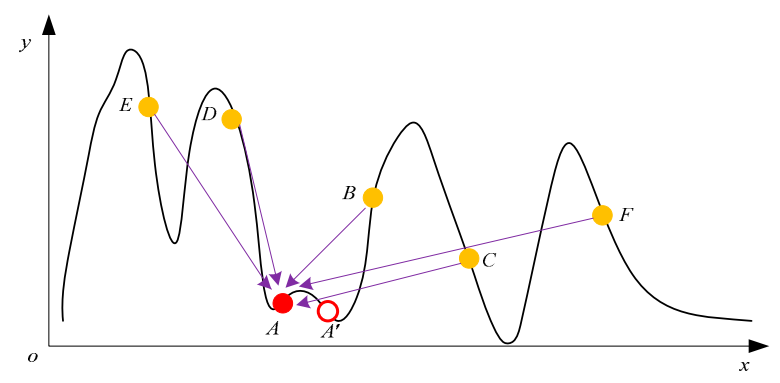

Figure 1. Particle movement in the basic grey wolf optimization (GWO) algorithms.

In the case of solving the minimum value that is shown in Figure 1, when A is the globally optimal particle, the search with only global guidance leads the particles with poor fitness to gather around $A$ rather than $A^{\prime}$ and easily fall into the local optimum. The main reason for this phenomenon is the lack of a further in-depth search for the neighborhood of the globally optimal particle $A$. Therefore, this paper adds the local search strategy to this algorithm and proposed an improved version of the GWO, namely, GWOI to improve the search ability of the standard GWO algorithm. As depicted in Figure 2, after adding the local search strategy into the standard GWO algorithm, GWOI can search the local neighborhood of the global optimal point $A$ in depth and it can easily search for a better candidate $A^{\prime}$. The direct method for enhancing its local search ability is to replace the alpha, beta, and delta with the first three optimal individuals of the current iteration in its encircling step formulas, since the GWO algorithm search is mainly controlled by the first three best wolves.

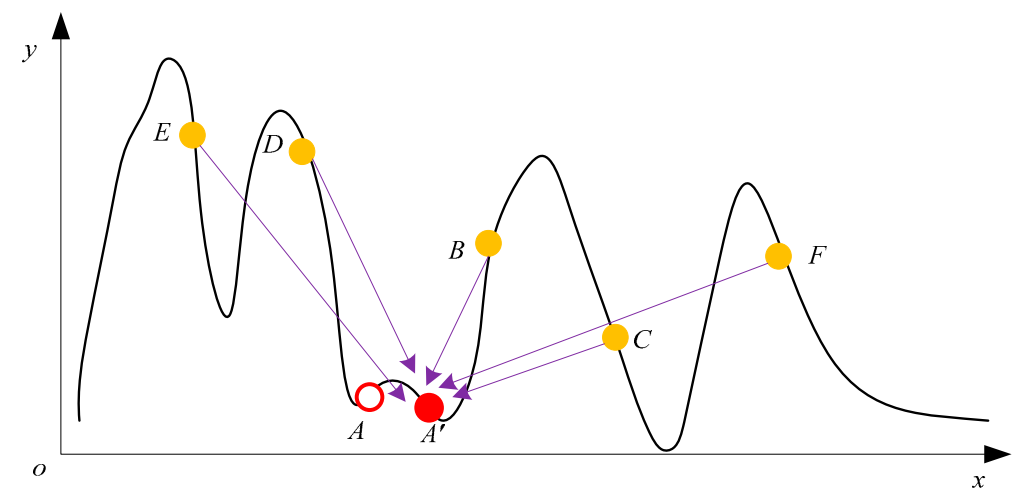

Figure 2. Particle movement in the GWOI.

The improved encircling step formulas are as follows:

$$
\begin{aligned}
& \vec{D}_{\alpha}^{\prime}=\left|\vec{C}_{1} \cdot \vec{X}_{\alpha}^{\prime}-\vec{X}\right| \\
& \vec{D}_{\beta}^{\prime}=\left|\begin{array}{l}
\vec{C}_{2} \cdot \vec{X}_{\beta}^{\prime}-\vec{X} \\
\vec{D}_{\delta}=\left|\vec{C}_{3} \cdot \vec{X}_{\delta}-\vec{X}\right|
\end{array}\right|
\end{aligned}
$$

where $\vec{X}_{\alpha}^{\prime}, \vec{X}_{\beta}^{\prime}$, and $\vec{X}^{\prime}$ are the first three best locally optimal individuals.

Therefore, the mathematical models of the search agents update their positions, as follows:

$$
\begin{aligned}
& \vec{X}_{1}^{\prime}=\vec{X}_{\alpha}^{\prime}-\vec{A}_{1} \cdot \vec{D}^{\prime}{ }_{\alpha} \\
& \vec{X}^{\prime}{ }^{2}=\vec{X}_{\beta}^{\prime}-\vec{A}_{2} \cdot \vec{D}^{\prime} \\
& \vec{X}_{\beta}^{\prime}=\vec{X}_{\delta}^{\prime}-\vec{A}_{3} \cdot \vec{D}^{\prime}{ }_{\delta}
\end{aligned}
$$




$$
\vec{X}(t+1)=\frac{\vec{X}_{1}^{\prime}+\vec{X}_{2}^{\prime}+\vec{X}_{3}^{\prime}}{3}
$$

\subsection{The Compared GWOII Algorithm}

Although the local search strategy is added to the GWOI algorithm to enhance its search ability, it only focuses on the local neighborhood search of the first three best particles. Once the globally optimal particles fall into the local optimum, the algorithm loses the ability to jump out of the local optimum, as shown in Figure 2. If the GWO algorithm can conduct an independent local search in the neighborhood of particles with similar fitness to the current optimal fitness, such as points $B, C$, $D, E$, and $F$, the probability of finding a better solution will greatly increase. This paper proposes a noninferior solution neighborhood independent local search technique based on this analysis. The main idea of this method is as follows: if the fitness value error between some particles and the current optimal particles is small, those particles are considered to be a noninferior solution and will no longer move toward the global optimal particle, but conduct a local depth search in their neighborhood to find a better solution that may exist. Figure 3 shows this search situation. In Figure 3, after introducing the noninferior solution neighborhood independent local search strategy and carrying out a certain number of iterative operations, points $B, C, D, E$, and $F$ find better points $B^{\prime}, C^{\prime}, D^{\prime}, E^{\prime}$, and $F^{\prime}$.

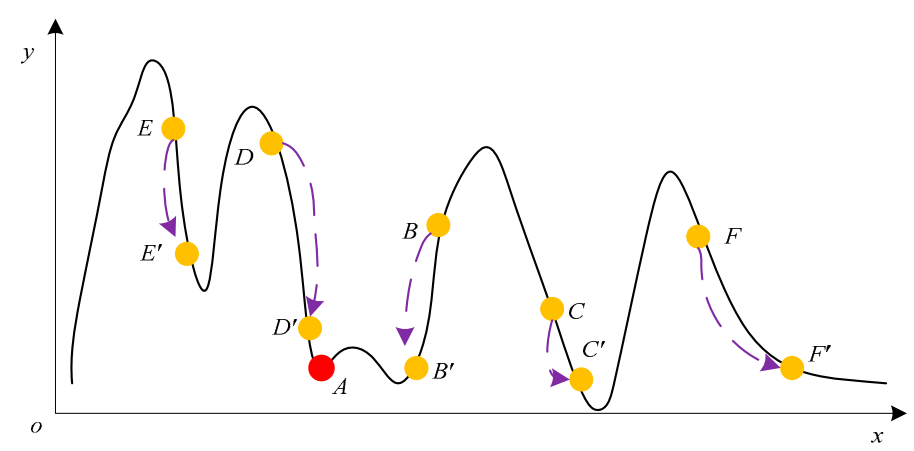

Figure 3. Particle movement under the guidance of the noninferior solution neighborhood independent local search strategy.

To implement the noninferior solution neighborhood independent local search technique, the determination conditions of the noninferior solution are as follows [37]:

$$
\begin{gathered}
F b_{i}(t)-F_{\text {best }}(t)<\lambda\left[\bar{F} b(t)-F_{\text {best }}(t)\right] \\
\lambda=\log _{0.5}(-t / T)
\end{gathered}
$$

where $\lambda$ is the adjustment parameter, $F b_{i}(t), F_{\text {best }}(t)$, and $\bar{F} b(t)$ represents the best fitness value recorded by the $i$ th particle in the $i$ th iteration, the best fitness value searched by the algorithm so far, and the average value of the optimal fitness value searched by each particle, respectively. If the algorithm satisfies the determination condition of Equation (9) during the iteration, then the corresponding particle is a noninferior solution; then, in the $(t+1)$ th iteration, the noninferior solution neighborhood independent local search method is executed, and its mathematical expression is as follows:

$$
\vec{X}_{i}(t+1)=\operatorname{pbest}_{i}(t)+\text { Cauchy }(t) \cdot\left(u b_{i}-l b_{i}\right) \cdot \exp \left(-2 \cdot \frac{t}{T} \cdot \pi\right) \cdot \cos \left(\pi \cdot \frac{t}{T}\right)
$$

where $\operatorname{pbest}_{i}(t)$ is the best position of the $i$ th particle obtained so far, Cauchy $(t)$ is the Cauchy random number in the th iteration and the reason that we chose this parameter is that it has better stability than the standard normal uniform distribution and is more conducive to the exploration of the algorithms, 
$u b_{i}$ and $l b_{i}$ are the $i$ th upper boundary and $i$ th lower boundary, respectively, of the search space, and $T$ represents the maximum number of iterations.

Therefore, the GWOII algorithm is proposed by introducing the noninferior solution independent local search strategy into the standard GWO algorithm. As described in Figure 4, this strategy enables the GWOII algorithm to find a better $C^{\prime}$ in the neighborhood of noninferior solution $C$. If the fitness value of $C^{\prime}$ is better than that of the global optimal point $A$, then the particles in the population will no longer move toward $A$, but toward $C^{\prime}$.

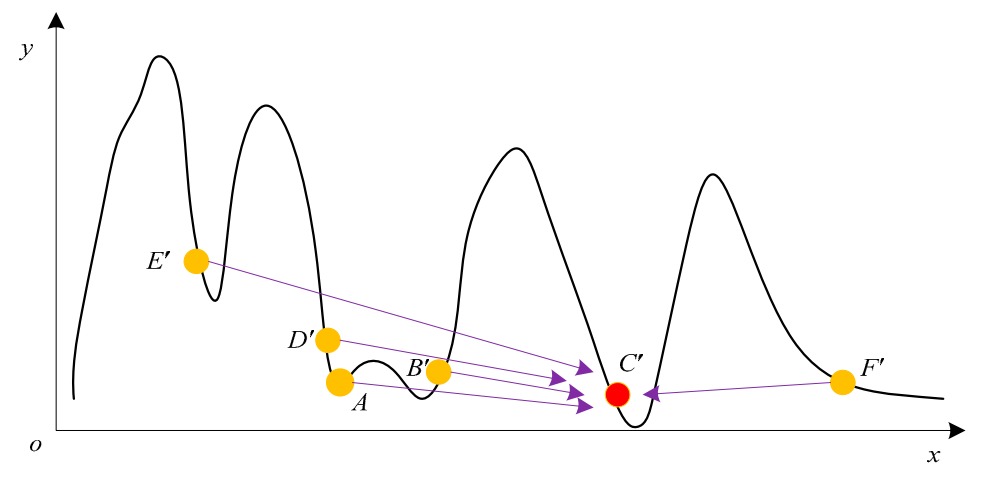

Figure 4. Particle movement in the GWOII.

\subsection{The Proposed NGWO Algorithm}

In this subsection, the GWOI algorithm is combined with the GWOII algorithm to form the proposed NGWO algorithm. The NGWO algorithm not only retains the strong search performance of the GWOI algorithm, but it also has the strong ability to jump out of the local optimal solution, as in the GWOII algorithm. Figure 5 shows the superiority of NGWO over GWO, GWOI, and GWOII in terms of optimization performance under certain optimization conditions.

In Figure 5, the local depth search method is used to search the neighborhood of the global optimal particle $A$ and to then find a better potential solution $A^{\prime}$; the noninferior solution neighborhood independent local search technique is adapted to search the noninferior solution $B, C, D, E$, and $F$, and better particles $B^{\prime}, C^{\prime}, D^{\prime}, E^{\prime}$, and $F^{\prime}$ are found. Moreover, among these better particles, the fitness of $C^{\prime}$ is better than that of the global optimal particle $A^{\prime}$. Therefore, the particles in the population move toward particle $C^{\prime}$ instead of toward particle $A^{\prime}$, and particle $C^{\prime}$ becomes the globally optimal particle.

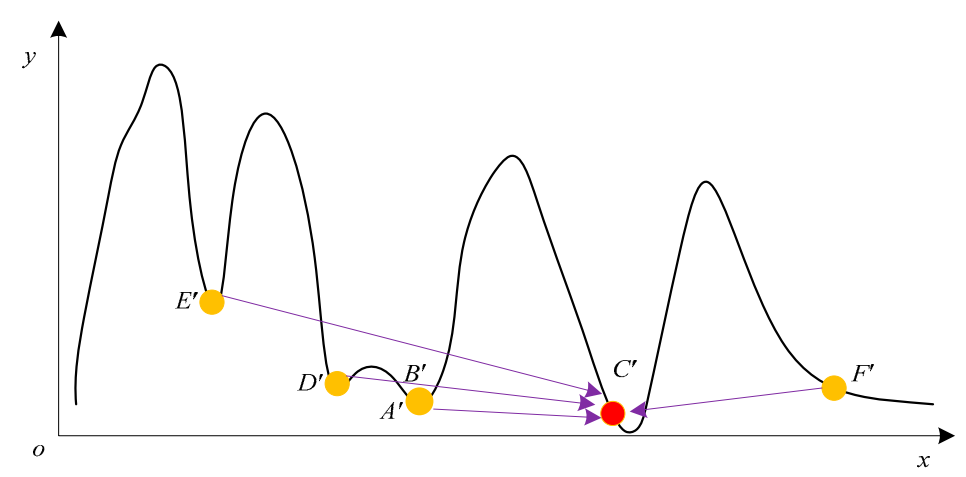

Figure 5. Particle movement in the novel GWO (NGWO) algorithms. 
Figure 6 details the flowchart of the proposed NGWO algorithm, and Algorithm 1 presents the pseudocode of the proposed NGWO.

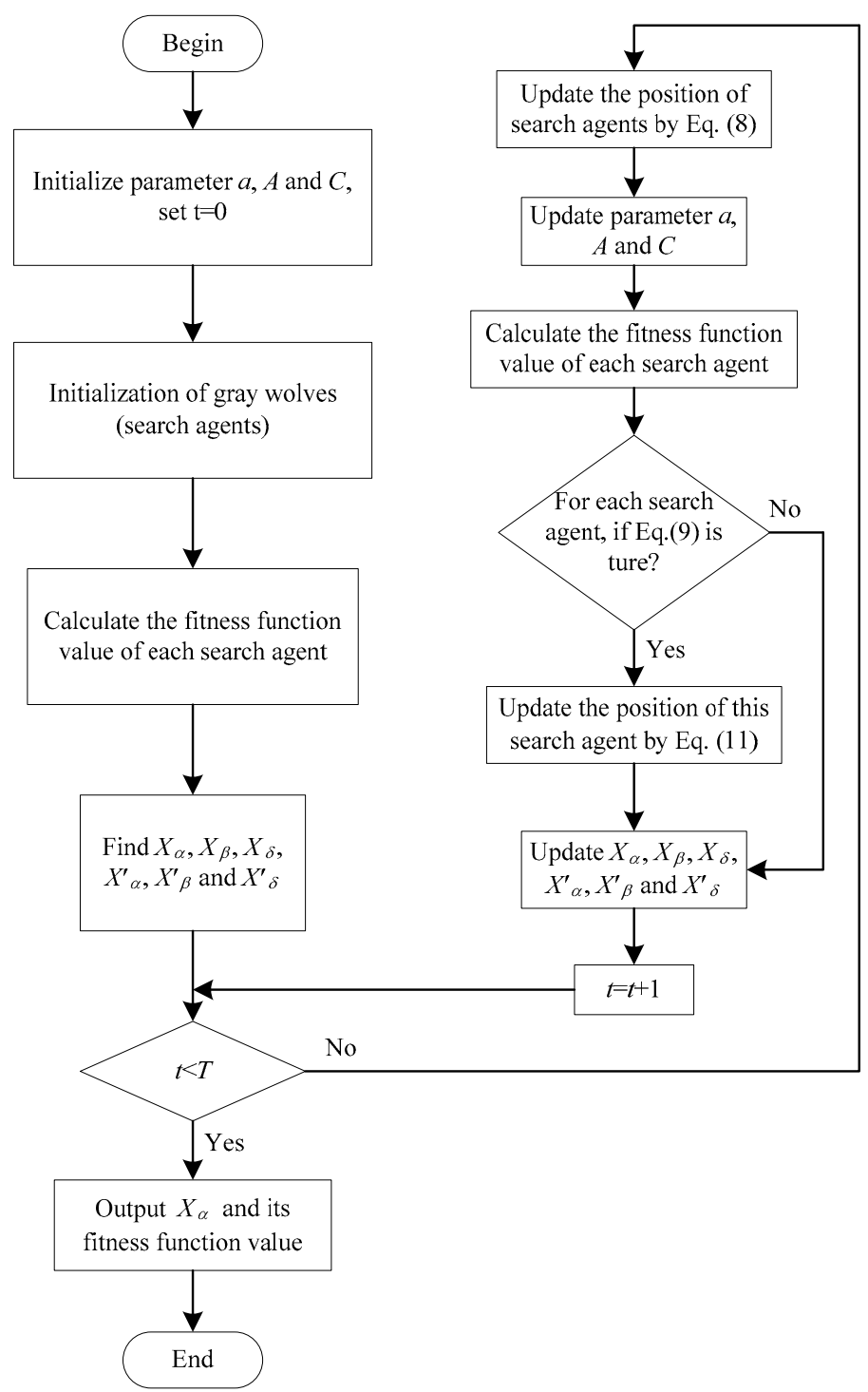

Figure 6. Flow chart of the proposed NGWO algorithm. 


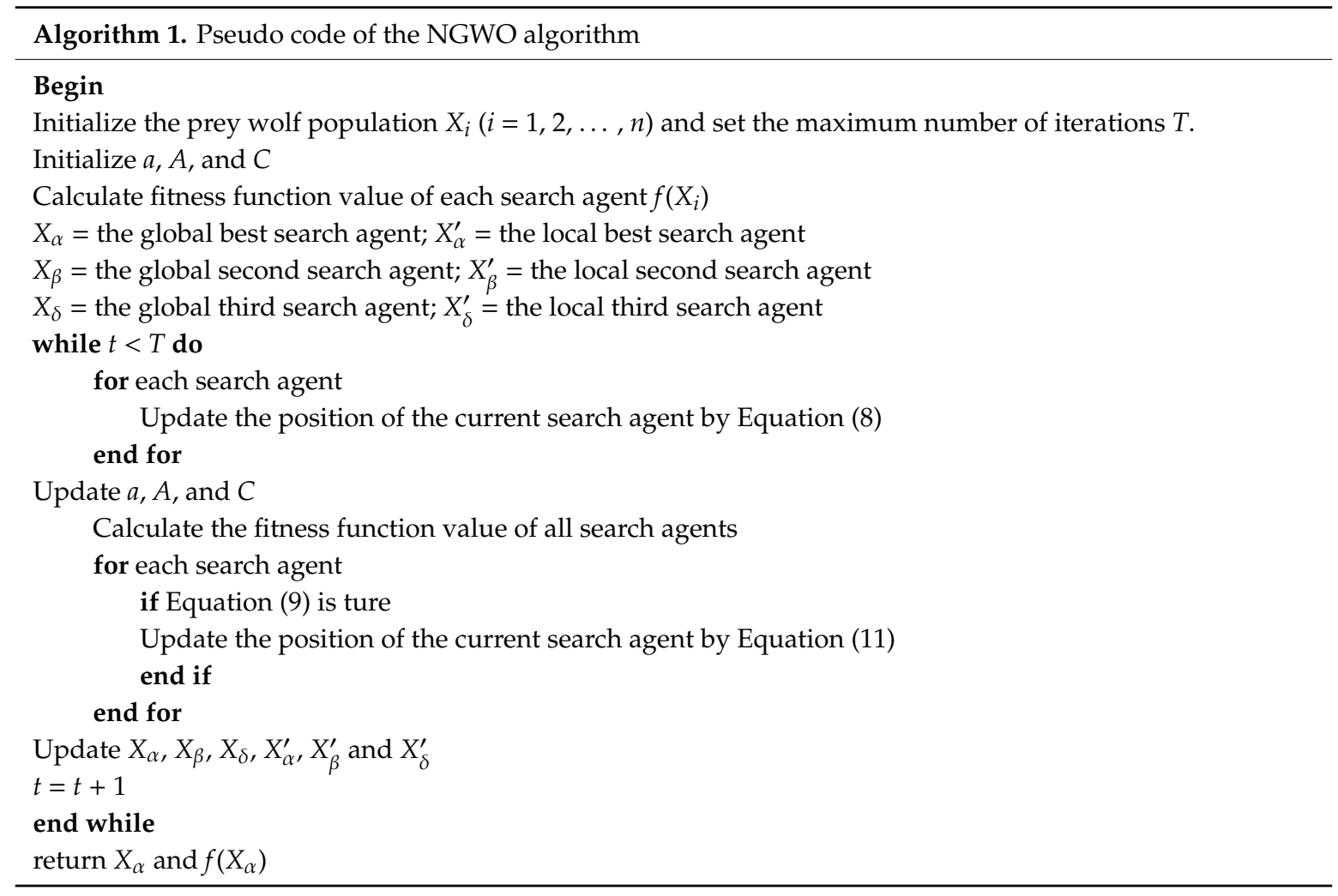

\section{Economic Load Dispatch Formulations}

The ELD problem can be described as an optimization problem to minimize the total fuel cost of the individual dispatchable generating power while being subject to different constraints. We adopt the problem descriptions and formulations from refs. [38,39].

\subsection{Objective Function}

The ELD problem sums all the costs of the committed generators. Mathematically, this problem can be modeled in Equation (12), as:

$$
\begin{gathered}
F=\sum_{i=1}^{n} F_{i}\left(P_{i}\right) \\
F_{i}\left(P_{i}\right)=a_{i} P_{i}^{2}+b_{i} P_{i}+c_{i}
\end{gathered}
$$

where $F$ is the total cost function of $n$ committed generating units, $F_{i}\left(P_{i}\right)$ is the generating cost function of the $i$ th generator with the generation output $P_{i}$, and $a_{i}, b_{i}$, and $c_{i}$ are the smooth cost fuel coefficients of the $i$ th generator, which are constants.

In real-life, the valve-point loading effects are modeled by adding a higher-order nonlinearity rectified sinusoid contribution to the power generating systems and they are represented using Equation (13), as follows:

$$
F_{i}\left(P_{i}\right)=a_{i} P_{i}^{2}+b_{i} P_{i}+c_{i}+\left|e_{i} \times \sin \left(f_{i} \times\left(P_{i}^{\min }-P_{i}\right)\right)\right|
$$

where $e_{i}$ and $f_{i}$ represent the nonsmooth cost fuel coefficients of the $i$ th generator; and, $P_{i}^{\text {min }}$ is the minimum generating capacity of the $i$ th generator. 
According to the description above, the objective function of the ELD problem with the valve-point effect can be formulated as:

$$
\min F=\sum_{i=1}^{n}\left(a_{i} P_{i}^{2}+b_{i} P_{i}+c_{i}+\left|e_{i} \times \sin \left(f_{i} \times\left(P_{i}^{\min }-P_{i}\right)\right)\right|\right)
$$

\subsection{Constraints and Variables}

The constraints and variables of Equation (15) are listed, as follows [40-43]:

\subsubsection{Power Balance Constraints and Variables}

The whole power demand must equal to the total power generated by available units minus the total transmission loss, which can be modeled as:

$$
\sum_{i=1}^{n} P_{i}-P_{\mathrm{loss}}=P_{\mathrm{demand}}
$$

where $P_{\text {loss }}$ and $P_{\text {demand }}$ are the value of power demand and whole transmission loss, respectively, in the system. Generally, $P_{\text {loss }}$ is calculated by Kron's loss formula, as shown in Equation (17).

$$
P_{\text {loss }}=\sum_{i=1}^{n} \sum_{j=1}^{n} P_{i} B_{i j} P_{j}+\sum_{i=1}^{n} B_{i 0} P_{i}+B_{00}
$$

where $B_{i j}, B_{i 0}$, and $B_{00}$ are the loss coefficients, which are assumed to be constants under normal circumstances.

\subsubsection{Generating Capacity Limits and Variables}

The actual output $P_{i}$ that is generated by the $i$ th available unit should range between its minimum generation capacity and maximum generation capacity:

$$
P_{i}^{\min } \leq P_{i} \leq P_{i}^{\max }
$$

where $P_{i}^{\min }$ and $P_{i}^{\max }$ are the minimum and maximum generating capacity of the $i$ th generator, respectively.

\subsubsection{Ramp Rate Limits and Variables}

In real circumstances, the operating range of each unit is restricted by its ramp-rate limit constraint:

$$
\max \left(P_{i}^{\min }, P_{i}^{0}-D R_{i}\right) \leq P_{i} \leq \min \left(P_{i}^{\max }, P_{i}^{0}+U R_{i}\right)
$$

where $P$ and $P_{i}^{0}$ are the current and previous power output, respectively, and $U R_{i}$ and $D R_{i}$ are the ramp-up and ramp-down limits of generator $i$, respectively.

\subsubsection{Prohibited Operating Zones Constraints and Variables}

In the actual situation, the valve-point loading effects affect the power system, and each generator contains some discontinuous POZs where the generator cannot work. Therefore, the feasible operating zones of each unit should be avoided in these prohibited zones and they can be demonstrated, as follows:

$$
\begin{gathered}
P_{i}^{\text {min }} \leq P_{i} \leq P_{i, 1}^{\text {lower }} \\
P_{i, j-1}^{\text {upper }} \leq P_{i} \leq P_{i, j}^{\text {lower }} \\
P_{i, n_{i}}^{\text {upper }} \leq P_{i} \leq P_{i}^{\max }
\end{gathered}
$$


where $P_{i, j}^{\text {lower }}$ and $P_{i, j}^{\text {upper }}$ are the lower and upper bounds, respectively, of the $j$ th POZs of the $i$ th generating unit, where $j \in\left[1, n_{i}\right]$, and $n_{i}$ is the total number of POZs of unit number $i$.

\section{Implementation of NGWO Method in Solving the ELD Problem}

In this subsection, the connection between the NGWO algorithm and the ELD problem was developed to obtain an efficient and high-quality solution. The NGWO algorithm was primarily employed to determine the optimal power generation for each unit that was operational during a particular period to minimize the total power generation cost. Two following definitions should be described in detail before using the proposed NGWO method to solve the ELD problem.

\subsection{Constraints Handling in ELD Problems with NGWO Approache}

The key point in applying the NGWO method to optimizing the ELD problem is how the NGWO algorithm handles the constraints that exist in the problem. In general, most of the researchers are more likely to employ the penalty function methods to treat the constrained optimization problems [44]. The introduction of a penalty function can transform a constrained problem into an unconstrained problem and build a single objective function, so using an unconstrained optimization method can minimize it. When using the NGWO algorithm to solve a constrained ELD problem, it is common to handle constraints using principles of penalty functions, as follows [44]:

$$
\min f= \begin{cases}f\left(P_{i}\right), & \text { if } P_{i} \in F l \\ f\left(P_{i}\right)+\text { penalty }\left(P_{i}\right), & \text { otherwise }\end{cases}
$$

where, penalty $\left(P_{i}\right)$ is 0 if no constraint is violated; otherwise it is positive value, $F l$ indicates the feasible region.

\subsubsection{ELD Problem without the Valve-Point Loading Effects}

In our work, when using the NGWO algorithm to handle the ELD problem without considering the valve-point loading effects, the map methods is built, as in Equation (22).

$$
P_{i}^{j}(t+1)=P_{i}^{\min }+x_{i}\left(P_{i}^{\max }-P_{i}^{\min }\right)
$$

where, $x_{i}$ is a value between 0 and 1 obtained by the NGWO method, and the meanings of $P_{i}^{\min }$ and $P_{i}^{\max }$ are shown in Section 3.2.

After establishing the map method, Equation (13) is rewritten as:

$$
\min F=\sum_{i=1}^{n} F_{i}\left(P_{i}\right) \cdot\left(1-q \cdot \max \left(\sum_{i=1}^{n} P_{i}-P_{\text {demand }}, 0\right)\right)
$$

where, $q$ is a positive constants (penalty factors).

\subsubsection{ELD Problem with Considering the Valve-Point Loading Effects}

In this article, the map method used for handling the valve-point loading effects in NGWO approaches is according to Equations (22)-(24), as follows:

$$
\begin{gathered}
P_{\text {lower }, i}=\max \left(P_{i}^{\min }, P_{i}^{0}-D R_{i}\right) \\
P_{\text {upper }, i}=\min \left(P_{i}^{\max }, P_{i}^{0}+U R_{i}\right) \\
P_{i}^{j}(t+1)=P_{\text {lower }, i}+x_{i} \cdot\left(P_{\text {upper }, i}-P_{\text {lower }, i}\right)
\end{gathered}
$$


where, $t$ is the current iteration, the meanings of $P_{i}^{\min }, P_{i}^{\max }, P_{i}^{0}, D R_{i}$, and $U R_{i}$ are shown in Section 3.2, $x_{i}$ is a value between 0 and 1 that is obtained by the NGWO method.

Next, if the equality Equation (16) and inequality Equations (19) and (20) are not solved, then Equation (15) is rewritten as:

$$
\min F=\sum_{i=1}^{n} F_{i}\left(P_{i}\right)+q \cdot \max \left(1-\frac{\sum_{i=1}^{n} P_{i}-P_{\text {loss }}}{P_{\text {demand }}}, 0\right)
$$

where, $q$ is a positive constant (penalty factors), the meanings of $P_{\text {loss }}$ and $P_{\text {demand }}$ are shown in Section 3.2.

\subsection{Implementation Steps of NGWO to ELD Problem}

This work presents a quick solution to the ELD problem while utilizing the NGWO algorithm to obtain global optimal or near global optimal generation quantity of each generator unit. The development steps of the proposed technique to solve the ELD problem were detailed, as below.

Step 1: Initialize the population size $N$ and the maximum number of iteration $T$, and randomly generate the grey individuals of the population between 0 and 1 .

Step 2: Calculate the fitness value.

Step 2.1 If it is the ELD problem without the valve-point loading effects, map these initialized grey wolf individuals to the feasible domain of the practical operation constraints according to Equation (22). Calculate the total cost function of $n$ committed generating units by using Equation (23) as the fitness value.

Step 2.2 If the valve-point loading effects are considered in the ELD problem, then map these initialized grey wolf individuals to the feasible domain of the practical operation constraints according to Equation (26), and employ the loss coefficients $B, B_{0}$, and $B_{00}$ to calculate the transmission loss $P_{\text {loss }}$ while using Equation (17). Calculate the total cost function of $n$ committed generation units using Equation (27) as the fitness value.

Step 3: Compare each individual's fitness value to find out $X_{a}, X_{\beta}, X_{\delta}, X_{\alpha}^{\prime}, X_{\beta}^{\prime}$, and $X_{\delta}^{\prime}$.

Step 4: Update the position of the current search agent by Equation (8).

Step 5: Update parameters $a, A$, and $C$.

Step 6: Calculate Equation (9). If Equation (9) is satisfied, then update the position of the current search agent by Equation (11).

Step 7: Use Step 2 to calculate fitness function value for each individual search agents.

Step 8: Update $X_{\alpha}, X_{\beta}, X_{\delta}, X_{\alpha}^{\prime}, X_{\beta}^{\prime}$, and $X_{\delta}^{\prime}$.

Step 9: If the number of iterations $t$ reaches the maximum $T$, then go to Step 10. Otherwise, go to Step 3.

Step 10: The latest generated individual $X_{\alpha}$ is the optimal and then maps $X_{\alpha}$ according to Step 2 to obtain $P\left(X_{\alpha}\right) . P\left(X_{\alpha}\right)$ is the optimal generation power of each unit, and its fitness value $F\left(P\left(X_{\alpha}\right)\right)$ is the minimum total generation cost.

Based on above analysis, the pseudocode of the NGWO algorithm employed to solve the ELD problem is shown in Algorithm 2. 


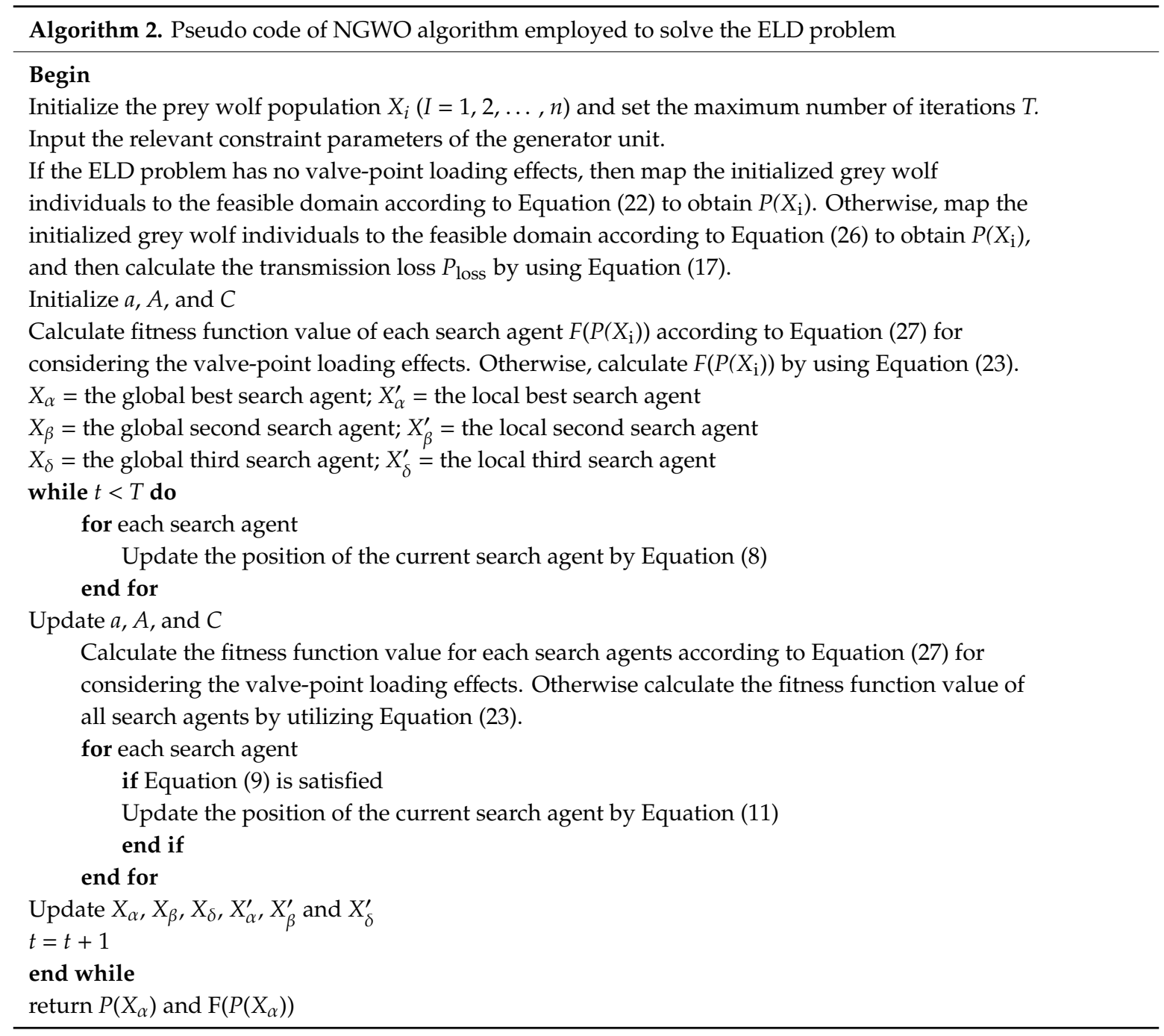

\section{Numerical Simulation Results and Analysis}

To verify the applicability of NGWO for solving the ELD problem, the performance of the basic GWO, the compared GWOI, the compared GWOII, and the proposed NGWO algorithms are assessed on the following ELD cases:

Case I. A 3-generator system for load demand of $850 \mathrm{MW}$, and valve-point loading effects are considered.

Case II. A 13-generator system for a load demand of $2520 \mathrm{MW}$, and valve-point loading effects are considered.

Case III. A 40-generator system for a load demand of $10500 \mathrm{MW}$, and valve-point loading effects are considered.

Case IV. A 6-generator system with a quadratic cost function, POZs and transmission loss, and a load demand of $1263 \mathrm{MW}$.

Case V. A 15-generator system with a quadratic cost function, POZs and transmission loss, and a load demand of $2630 \mathrm{MW}$.

In this paper, the parameters set for each case study mentioned above are listed below. Each optimization technique was coded in MATLAB 2015a and executed on a Windows 10, 4-GHz, 2-GB RAM processor. In addition, the numbers of 50 independent runs were recorded for the compared GWOI, GWOII, and the proposed NGWO algorithms to validate the robustness of the proposed 
optimization technique. Furthermore, the population size was set to 30 for each case. Finally, for each ELD case, the maximum number of iterations was set to 500 .

\subsection{Case I: 3-Generator System}

This case study consists of three generating units with quadratic cost functions and the effects of valve-point loadings are considered [45]. Table 1 provides the data of the generating units. In this case study, NGWO is compared with GWO, GWOI, GWOII, GA, and PSO [46], CJAYA and MP-CJAYA [37], and EP [47], in terms of the mean $\left(F_{\text {mean }}\right)$ and the best $\left(F_{\text {best }}\right)$ total generation cost. Table 2 records the comparison results. Figure 7 shows the convergence curve of the total generation cost for the mean solution, and Figure 8 explicitly shows the robustness of GWO, GWOI, GWOII, and NGWO in 30 trials.

Table 1. Generating units' parameters for Case I with value-point loading.

\begin{tabular}{cccccccc}
\hline Generator & $\boldsymbol{P}_{\boldsymbol{i} \text { min }}(\mathbf{M W})$ & $\boldsymbol{P}_{\boldsymbol{i} \text { max }}(\mathbf{M W})$ & $\boldsymbol{a}_{\boldsymbol{i}}$ & $\boldsymbol{b}_{\boldsymbol{i}}$ & $\boldsymbol{c}_{\boldsymbol{i}}$ & $\boldsymbol{e}_{\boldsymbol{i}}$ & $\boldsymbol{f}_{\boldsymbol{i}}$ \\
\hline 1 & 100 & 600 & 0.001562 & 7.92 & 561 & 300 & 0.0315 \\
2 & 50 & 200 & 0.004820 & 7.97 & 78 & 150 & 0.063 \\
3 & 100 & 400 & 0.001940 & 7.85 & 310 & 200 & 0.042 \\
\hline
\end{tabular}

As shown in Table 2, GWO, GWOI, GWOII, and NGWO continuously decrease the values of $F_{\text {best }}$ and $F_{\text {mean }}$, and NGWO achieves the very competitive minimum value of $8223.104 \$ / \mathrm{h}$ relative to that of GA of $8222.07 \$ / \mathrm{h}$, as well as the very close minimum $F_{\text {mean }}$ value $8233.567 \$ / \mathrm{h}$ relative to that of MP-CJAYA of $8232.06 \$ / \mathrm{h}$. Therefore, the NGWO could obtain the second best results when compared to the above eight mentioned algorithms. The convergence curve in Figure 7 shows that the convergence rates of GWO, GWOI, GWOII, and NGWO continuously improved, and NGWO had the fastest convergence rate. Figure 8 confirms that NGWO achieved the best robustness.

Table 2. Best outputs of different methods for three-units system ( $\mathrm{PD}=850 \mathrm{MW})$.

\begin{tabular}{|c|c|c|c|c|c|c|c|c|c|}
\hline Unit & GA [46] & PSO [46] & MP-CJAYA [37] & CJAYA [37] & EP [47] & GWO & GWOI & GWOII & NGWO \\
\hline 2 & 399.600 & 400.000 & 400.000 & 400.000 & 400.000 & 399.600 & 399.600 & 399.600 & 399.600 \\
\hline$P_{\text {total }}(\mathrm{MW})$ & 848.400 & 850.000 & 850.004 & 849.977 & 850.000 & 850.011 & 850.021 & 850.010 & 850.005 \\
\hline$F_{\text {mean }}(\$ / h)$ & 8234.72 & 8234.09 & 8232.06 & 8289.41 & 8234.16 & 8305.91 & 8284.772 & 8240.313 & 8233.567 \\
\hline$F_{\text {best }}(\$ / h)$ & 8222.07 & 8234.07 & 8223.29 & 8226.18 & 8234.07 & 8223.61 & 8223.367 & 8223.197 & 8223.104 \\
\hline
\end{tabular}

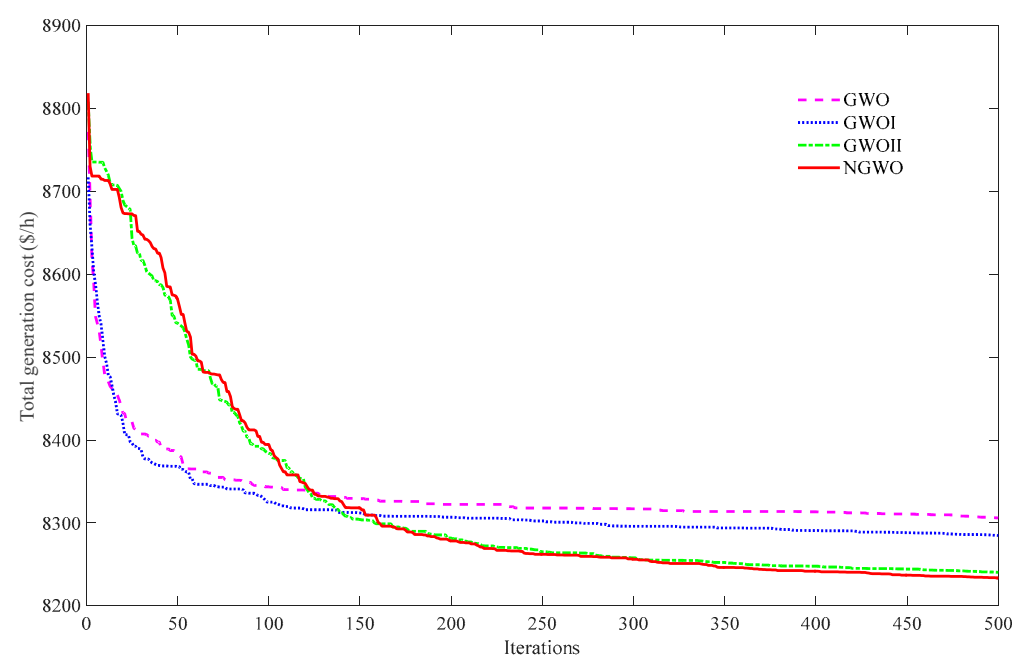

Figure 7. Convergence curve of the total generation cost for the mean solution in Case 1. 


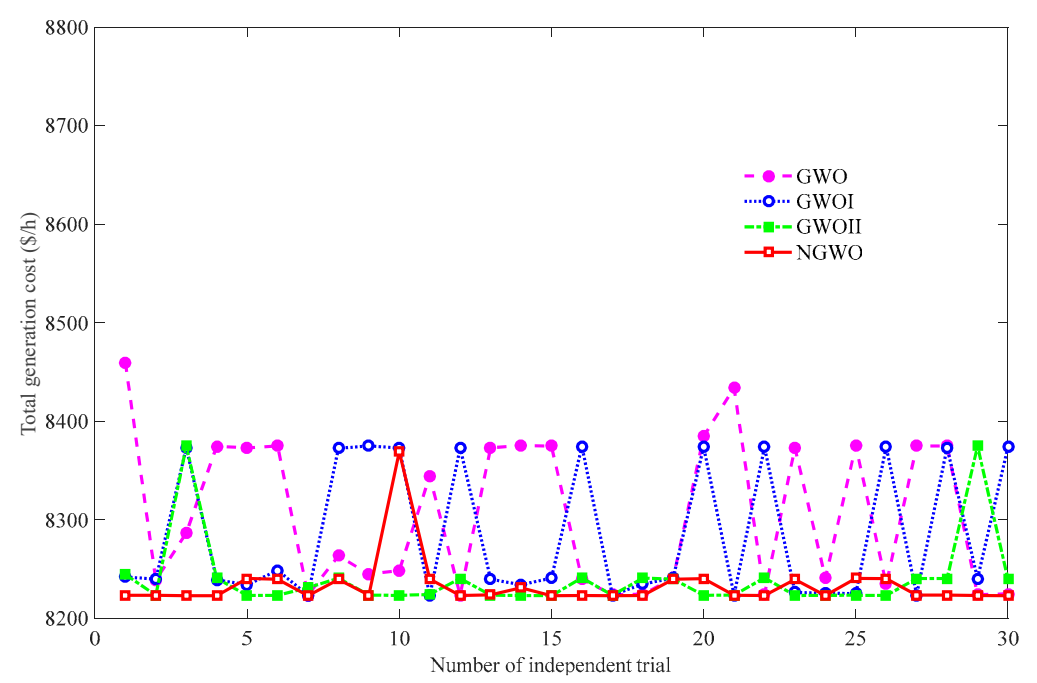

Figure 8. Total generation cost obtained by GWO, GWOI, GWOII, and NGWO for 30 trials in Case 1.

\subsection{Case II: 13-Generator System}

This case study is the second system with both valve-point loading effects and multiple fuel options and it comprises 13 generating units with quadratic cost functions. Table 3 shows all detailed data, which are taken from refs. [38,43]. The total power demand is $2520 \mathrm{MW}$. With an increasing number of generators, this system becomes more nonlinear and complex when compared to Case I. The results obtained by GWO, GWOI, GWOII, and NGWO were compared with GA [46], CPSO [48], JAYA and CJAYA [37], and SA [48], as listed in Table 4. The comparison results confirm that NGWO achieved the optimum total generation cost of both $F_{\text {mean }}$ and $F_{\text {best }}$ among the other algorithms, which were $24,366.12 \$ / h$ and $24,185.45 \$ / h$, respectively. Figure 9 shows the convergence curves of GWO, GWOI, GWOII, and NGWO for the mean value of the total generation cost in 30 trials. From Figure 9, GWO and GWOI have better convergence rates in the early iteration than GWOII and NGWO, but they easily fall into the local optimal solution, and GWOII and NGWO more easily obtain the global optimal solution in the later iteration. Figure 10 describes the distribution of the total generation cost of GWO, GWOI, GWOII, and NGWO in 30 trials. NGWO was more robust than GWO, GWOI, and GWOII.

Table 3. Generating units' parameters for Case II with value-point loading.

\begin{tabular}{cccccccc}
\hline Generator & $\boldsymbol{P}_{\boldsymbol{i} \text { min }}(\mathbf{M W})$ & $\boldsymbol{P}_{\boldsymbol{i} \text { max }}(\mathbf{M W})$ & $\boldsymbol{a}_{\boldsymbol{i}}$ & $\boldsymbol{b}_{\boldsymbol{i}}$ & $\boldsymbol{c}_{\boldsymbol{i}}$ & $\boldsymbol{e}_{\boldsymbol{i}}$ & $\boldsymbol{f}_{\boldsymbol{i}}$ \\
\hline 1 & 00 & 680 & 0.00028 & 8.10 & 550 & 300 & 0.035 \\
2 & 00 & 360 & 0.00056 & 8.10 & 309 & 200 & 0.042 \\
3 & 00 & 360 & 0.00056 & 8.10 & 307 & 200 & 0.042 \\
4 & 60 & 180 & 0.00324 & 7.74 & 240 & 150 & 0.063 \\
5 & 60 & 180 & 0.00324 & 7.74 & 240 & 150 & 0.063 \\
6 & 60 & 180 & 0.00324 & 7.74 & 240 & 150 & 0.063 \\
7 & 60 & 180 & 0.00324 & 7.74 & 240 & 150 & 0.063 \\
8 & 60 & 180 & 0.00324 & 7.74 & 240 & 150 & 0.063 \\
9 & 60 & 180 & 0.00324 & 7.74 & 240 & 150 & 0.063 \\
10 & 40 & 120 & 0.00284 & 8.6 & 126 & 100 & 0.084 \\
11 & 40 & 120 & 0.00284 & 8.6 & 126 & 100 & 0.084 \\
12 & 55 & 120 & 0.00284 & 8.6 & 126 & 100 & 0.084 \\
13 & 55 & 120 & 0.00284 & 8.6 & 126 & 100 & 0.084 \\
\hline
\end{tabular}


Table 4. Best outputs of different methods for 13-units system (PD = $2520 \mathrm{MW})$.

\begin{tabular}{|c|c|c|c|c|c|c|c|c|c|}
\hline Unit & GA [46] & CPSO [48] & CJAYA [37] & JAYA [37] & SA [46] & GWO & GWOI & GWOII & NGWO \\
\hline 1 & 627.05 & 628.32 & 628.3185 & 628.3185 & 668.40 & 647.3842 & 645.5569 & 630.9811 & 630.9951 \\
\hline 2 & 359.40 & 299.83 & 299.1992 & 299.2009 & 359.78 & 306.3995 & 306.9539 & 300.8038 & 297.9355 \\
\hline 3 & 358.95 & 299.17 & 299.1993 & 306.9105 & 358.20 & 309.6117 & 306.5356 & 302.7475 & 299.9253 \\
\hline 4 & 158.93 & 159.70 & 159.7330 & 159.7339 & 104.28 & 175.1400 & 169.6878 & 160.1702 & 157.9267 \\
\hline 5 & 159.73 & 159.64 & 159.7331 & 159.7337 & 60.36 & 66.8791 & 168.4922 & 161.0252 & 159.6433 \\
\hline 6 & 159.68 & 159.67 & 159.7331 & 159.7338 & 110.64 & 162.7466 & 174.9721 & 160.9845 & 159.2335 \\
\hline 9 & 110.15 & 159.78 & 159.7331 & 159.7340 & 161.52 & 175.1400 & 116.8800 & 159.7720 & 159.4265 \\
\hline 10 & 77.27 & 112.46 & 110.0403 & 114.8012 & 117.09 & 116.7600 & 116.8800 & 116.8577 & 76.8790 \\
\hline 11 & 75.00 & 74.00 & 114.7994 & 114.8001 & 75.00 & 116.7600 & 109.9096 & 77.0418 & 79.5038 \\
\hline 12 & 60.00 & 56.50 & 55.0000 & 92.4018 & 60.00 & 99.9167 & 59.0347 & 91.4990 & 86.8040 \\
\hline 13 & 55.41 & 91.64 & 55.0000 & 55.0027 & 119.58 & 108.5598 & 66.5129 & 88.6915 & 94.1941 \\
\hline$P_{\text {total }}(\mathrm{MW})$ & 2520 & 2520 & 2519.96 & 2519.97 & 2520 & 2520.83 & 2521.74 & 2520.13 & 2520.21 \\
\hline
\end{tabular}

" - " indicates the cost value is missing.

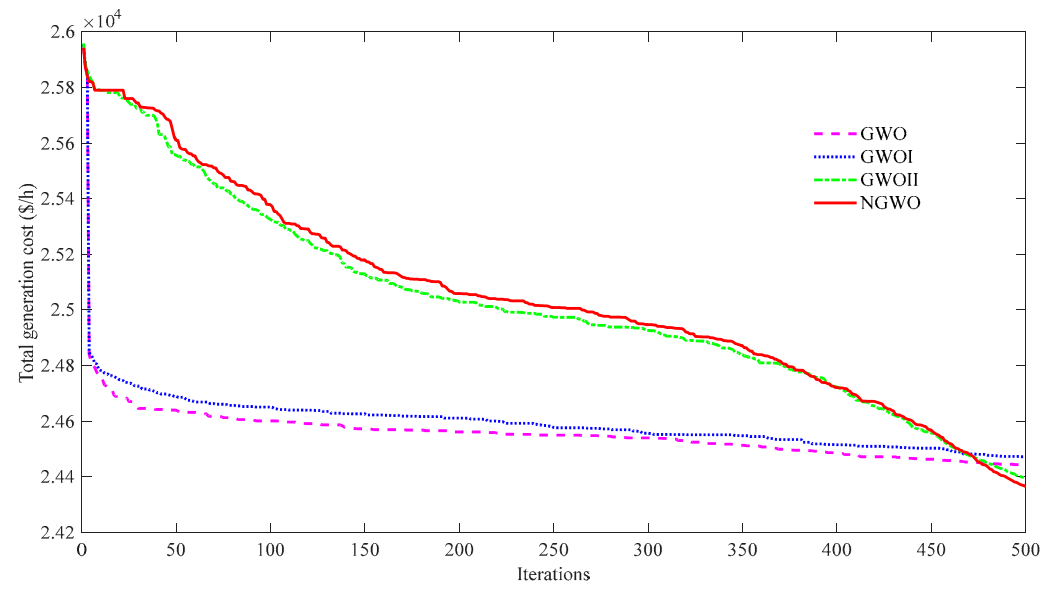

Figure 9. Convergence curve of the total generation cost for the mean solution in Case II.

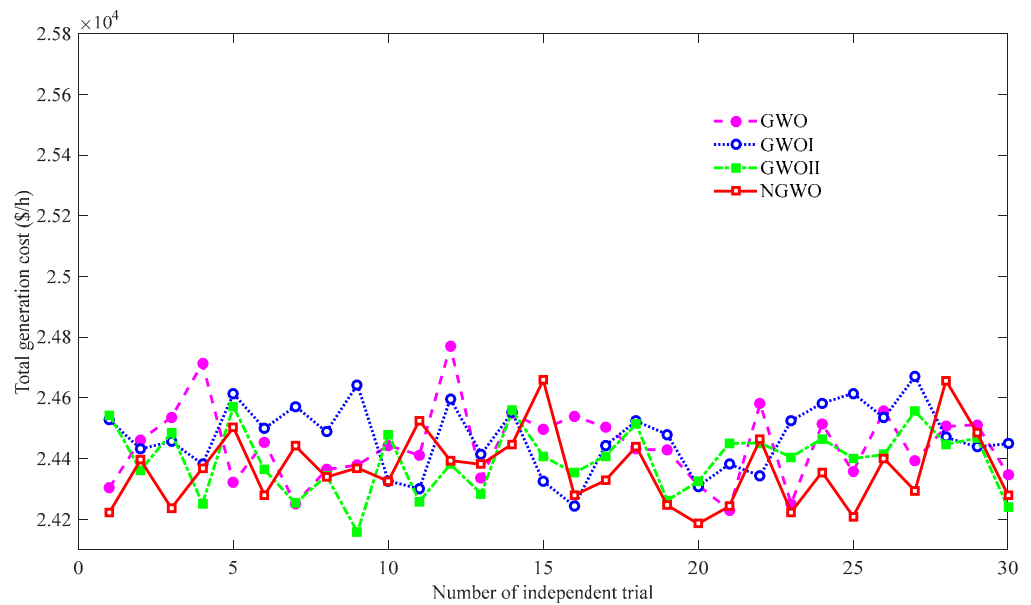

Figure 10. Total generation cost obtained by GWO, GWOI, GWOII, and NGWO for 30 trials in Case II.

\subsection{Case III: 40-Generator System}

In this subsection, the largest ELD problem system consisting of 40 generators, in which the valve-point effect is considered, with a total load demand of 10,500 MW, is selected to investigate the effectiveness of the NGWO algorithm. Table 5 provides the test data for this case study, in which the valve-point loading has also been included in the fuel cost functions [47]. Due to the large 
number of generator units in this test system, it has a much more complex solution when compared to the previous solution; therefore, the test system is very suitable for testing the difference in the optimization performance of different improvement strategies for the same algorithm. Table 6 reports the comparison results of the proposed NGWO method with NPSO [49], PSO-LRS [37], MPSO [13], CJAYA [37], IGA [50], GWO, GWOI, and GWOII. The table shows that NGWO solved the large-scale ELD problem with a high-quality optimum, and its optimization ability was slightly worse than that of CJAYA, but was better than that of the other methods. In these eight comparison algorithms, NGWO achieved the second optimization results. In Figure 11, the convergence curves of four different GWO versions are compared, and it can be observed that NGWO and GWOII dramatically accelerated the convergence rate. However, GWO and GWOI were easily trapped in the local optimum. Figure 12 is the distribution of the optimal total generation cost values that were obtained by GWO, GWOI, and GWOII in 30 runs. This figure demonstrates that these three algorithms show poor stability when optimizing this large-scale power system. However, the stability of the NGWO algorithm is relatively better. All of the above comparisons provide strong evidence demonstrating the effectiveness of NGWO in solving large-scale ELD problems.

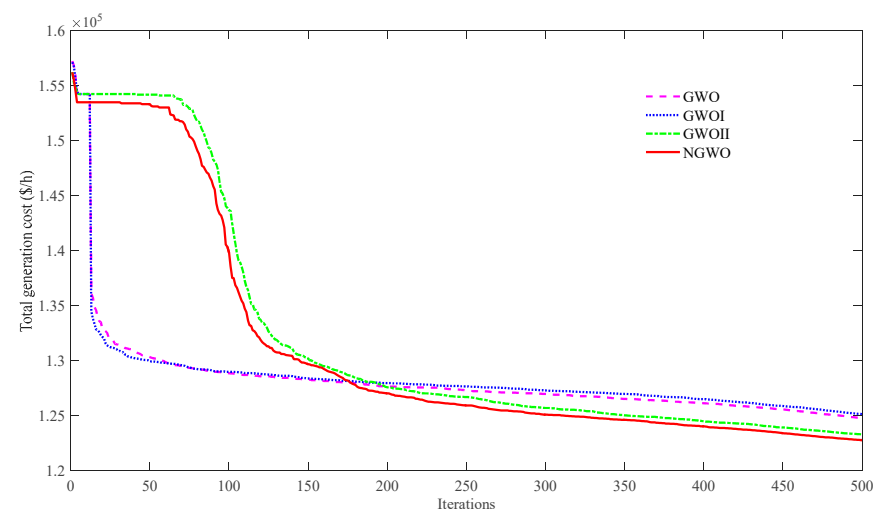

Figure 11. Convergence curve of total generation cost for the mean solution in Case III.

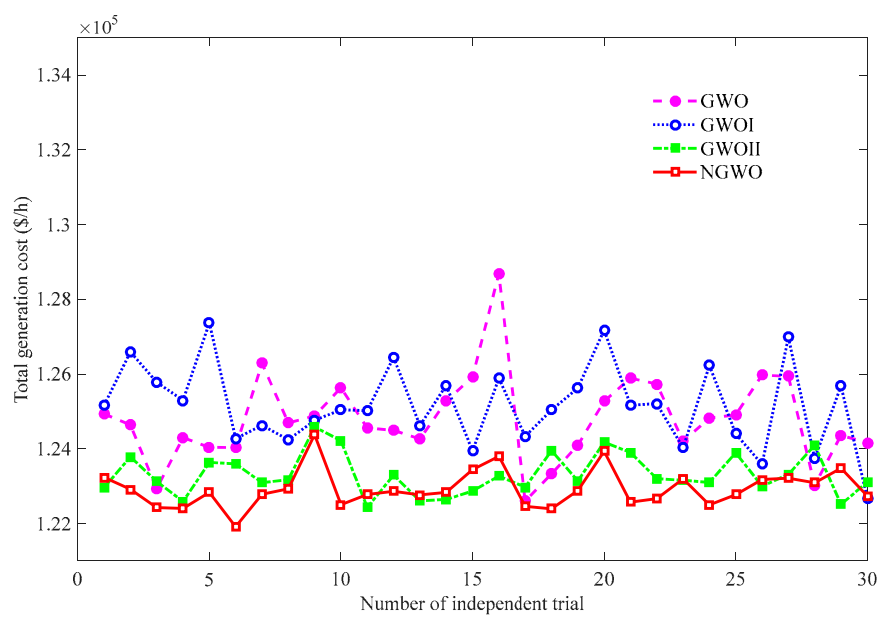

Figure 12. Total generation cost obtained by GWO, GWOI, GWOII, and NGWO for 30 trials in Case III. 
Table 5. Generating units' parameters for Case III with value-point loading.

\begin{tabular}{|c|c|c|c|c|c|c|c|}
\hline Generator & $P_{i \min }(\mathrm{MW})$ & $P_{i \max }(\mathrm{MW})$ & $a_{i}$ & $b_{i}$ & $c_{i}$ & $e_{i}$ & $f_{i}$ \\
\hline 1 & 36 & 114 & 0.00690 & 6.73 & 94.705 & 100 & 0.084 \\
\hline 2 & 36 & 114 & 0.00690 & 6.73 & 94.705 & 100 & 0.084 \\
\hline 3 & 60 & 120 & 0.02028 & 7.07 & 309.54 & 100 & 0.084 \\
\hline 4 & 80 & 190 & 0.00942 & 8.18 & 369.03 & 150 & 0.063 \\
\hline 5 & 46 & 97 & 0.01140 & 5.35 & 148.89 & 120 & 0.077 \\
\hline 6 & 68 & 140 & 0.01142 & 8.05 & 222.33 & 100 & 0.084 \\
\hline 7 & 110 & 300 & 0.00357 & 8.03 & 287.71 & 200 & 0.042 \\
\hline 8 & 135 & 300 & 0.00492 & 6.99 & 391.98 & 200 & 0.042 \\
\hline 9 & 135 & 300 & 0.00573 & 6.60 & 455.76 & 200 & 0.042 \\
\hline 10 & 130 & 300 & 0.00606 & 12.9 & 722.82 & 200 & 0.042 \\
\hline 11 & 94 & 375 & 0.00515 & 12.9 & 635.20 & 200 & 0.042 \\
\hline 12 & 94 & 375 & 0.00569 & 12.8 & 654.69 & 200 & 0.042 \\
\hline 13 & 125 & 500 & 0.00421 & 12.5 & 913.40 & 300 & 0.035 \\
\hline 14 & 125 & 500 & 0.00752 & 8.84 & 1760.4 & 300 & 0.035 \\
\hline 15 & 125 & 500 & 0.00708 & 9.15 & 1728.3 & 300 & 0.035 \\
\hline 16 & 125 & 500 & 0.00708 & 9.15 & 1728.3 & 300 & 0.035 \\
\hline 17 & 220 & 500 & 0.00313 & 7.97 & 647.85 & 300 & 0.035 \\
\hline 18 & 220 & 500 & 0.00313 & 7.95 & 649.69 & 300 & 0.035 \\
\hline 19 & 242 & 550 & 0.00313 & 7.97 & 647.83 & 300 & 0.035 \\
\hline 20 & 242 & 550 & 0.00313 & 7.97 & 647.81 & 300 & 0.035 \\
\hline 21 & 254 & 550 & 0.00298 & 6.63 & 785.96 & 300 & 0.035 \\
\hline 22 & 254 & 550 & 0.00298 & 6.63 & 785.96 & 300 & 0.035 \\
\hline 23 & 254 & 550 & 0.00284 & 6.66 & 794.53 & 300 & 0.035 \\
\hline 24 & 254 & 550 & 0.00284 & 6.66 & 794.53 & 300 & 0.035 \\
\hline 25 & 254 & 550 & 0.00277 & 7.10 & 801.32 & 300 & 0.035 \\
\hline 26 & 254 & 550 & 0.00277 & 7.10 & 801.32 & 300 & 0.035 \\
\hline 27 & 10 & 150 & 0.52124 & 3.33 & 1055.1 & 120 & 0.077 \\
\hline 28 & 10 & 150 & 0.52124 & 3.33 & 1055.1 & 120 & 0.077 \\
\hline 29 & 10 & 150 & 0.52124 & 3.33 & 1055.1 & 120 & 0.077 \\
\hline 30 & 47 & 97 & 0.01140 & 5.35 & 148.89 & 120 & 0.077 \\
\hline 31 & 60 & 190 & 0.00160 & 6.43 & 222.92 & 150 & 0.063 \\
\hline 32 & 60 & 190 & 0.00160 & 6.43 & 222.92 & 150 & 0.063 \\
\hline 33 & 60 & 190 & 0.00160 & 6.43 & 222.92 & 150 & 0.063 \\
\hline 34 & 90 & 200 & 0.0001 & 8.95 & 107.87 & 200 & 0.042 \\
\hline 35 & 90 & 200 & 0.0001 & 8.62 & 116.58 & 200 & 0.042 \\
\hline 36 & 90 & 200 & 0.0001 & 8.62 & 116.58 & 200 & 0.042 \\
\hline 37 & 25 & 110 & 0.0161 & 5.88 & 307.45 & 80 & 0.098 \\
\hline 38 & 25 & 110 & 0.0161 & 5.88 & 307.45 & 80 & 0.098 \\
\hline 39 & 25 & 110 & 0.0161 & 5.88 & 307.45 & 80 & 0.098 \\
\hline 40 & 242 & 550 & 0.00313 & 7.97 & 647.83 & 300 & 0.035 \\
\hline
\end{tabular}


Table 6. Best outputs of different methods for 40-units system.

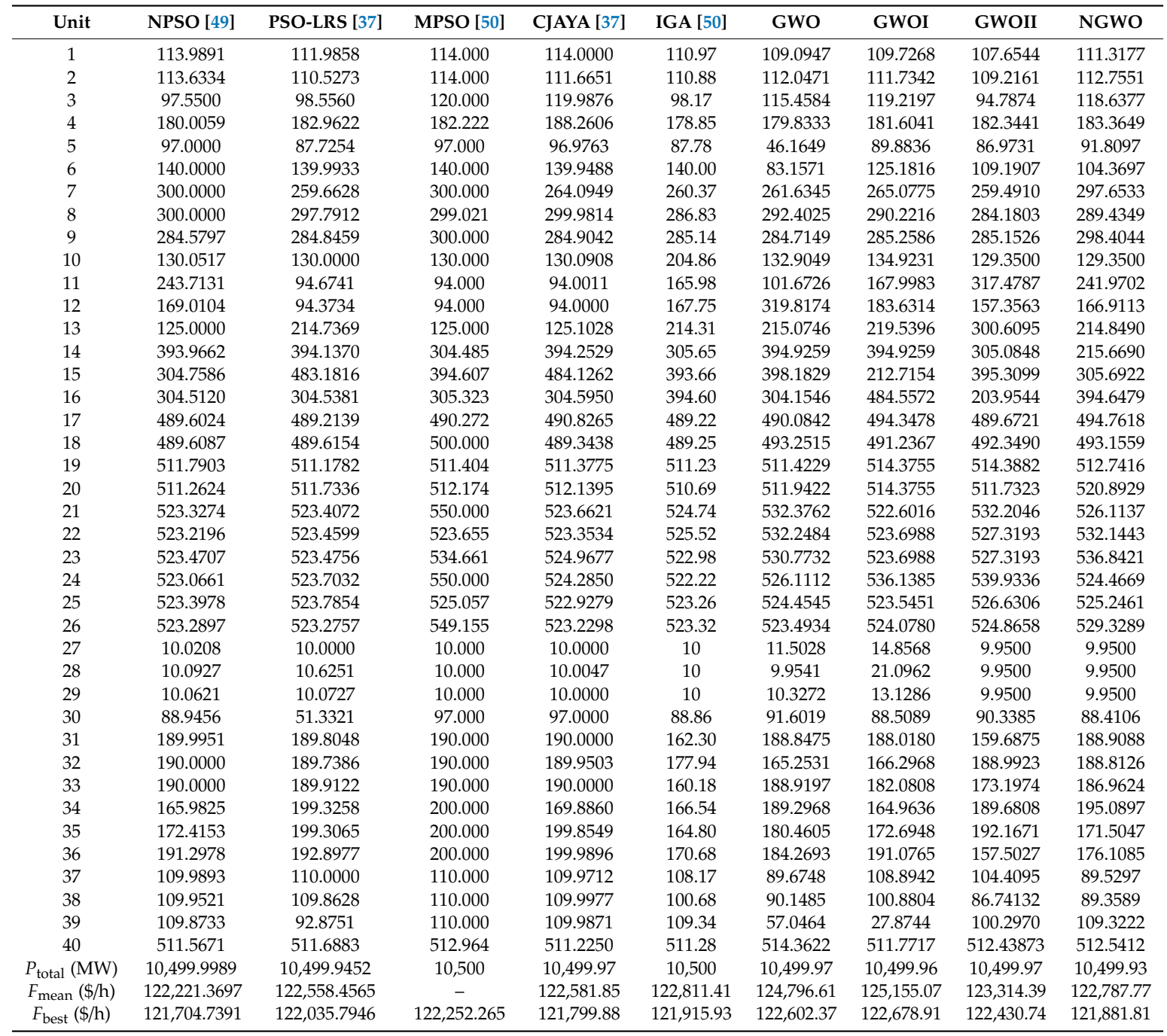

\subsection{Case IV: 6-Generator System}

This case study comprises six generating units with constraints of transmission loss, together with two POZs with ramp-up and ramp-down. The generator data and two POZs are recorded in Table 7, and Table 8 shows the loss B-coefficients [11]. The algorithm used to find the global optima for this problem always encounters challenging complexity, owing to the decision spaces being nonconvex and the cost functions being convex and represented by quadratic functions.

To validate the effectiveness of our method on this test system, NGWO is compared with SA [46], GA [51], MTS [52], NPSO [49], PSO [46], JAYA [37], GWO, GWOI, and GWOII in terms of the total generation cost. Table 9 provides the comparison confirming that GWOI obtained the lowest best total generation cost $\left(F_{\text {best }}\right)$ of $15,443.25 \$ /$ h among all of the techniques, while JAYA and NGWO achieved the second and third lowest $F_{\text {best }}$ of $15,447.09 \$ / \mathrm{h}$ and $15,449.17 \$ / \mathrm{h}$, respectively. In addition, Table 10 summarizes the best total generation cost $\left(F_{\text {best }}\right)$, worst total generation cost $\left(F_{\text {worst }}\right)$, and mean total generation cost $\left(F_{\text {mean }}\right)$ of the four versions of GWO. From Table 10, we can observe that NGWO provided the lowest $F_{\text {best }}$ and $F_{\text {mean }}$ of $15,449.17 \$ / \mathrm{h}$ and $15,449.86 \$ / \mathrm{h}$, respectively, and GWOII obtained the lowest $F_{\text {worst }}$ of $15,452.41 \$ / \mathrm{h}$. Figure 13 plots the distribution of the total generation cost for the mean solution, which shows that NGWO is the fastest among the four versions of GWO in terms of the convergence rate and it approaches the global optimum. In addition, NGWO has the most robust characteristics, as described in Figure 14. 
Table 7. Ramp rate limits and prohibited zones of units for Case IV.

\begin{tabular}{cccccccccc}
\hline Generator & $\boldsymbol{P}_{\boldsymbol{i} \text { min }}(\mathbf{M W})$ & $\boldsymbol{P}_{\boldsymbol{i} \text { max }}(\mathbf{M W})$ & $\boldsymbol{a}_{\boldsymbol{i}}$ & $\boldsymbol{b}_{\boldsymbol{i}}$ & $\boldsymbol{c}_{\boldsymbol{i}}$ & $\boldsymbol{P}_{\boldsymbol{i}}^{0}$ & $\boldsymbol{U R}_{\boldsymbol{i}}$ & $\boldsymbol{D R}_{\boldsymbol{i}}$ & Prohibited Zones \\
\hline 1 & 100 & 500 & 0.0070 & 7.0 & 240 & 440 & 80 & 120 & {$[210,240],[350,380]$} \\
2 & 50 & 200 & 0.0095 & 10.0 & 200 & 170 & 50 & 90 & {$[90,110],[140,160]$} \\
3 & 80 & 300 & 0.0090 & 8.5 & 220 & 200 & 65 & 100 & {$[150,170],[210,240]$} \\
4 & 50 & 150 & 0.0090 & 11.0 & 200 & 150 & 50 & 90 & {$[80,90],[110,120]$} \\
5 & 50 & 200 & 0.0080 & 10.5 & 220 & 190 & 50 & 90 & {$[90,110],[140,150]$} \\
6 & 50 & 120 & 0.0075 & 12.0 & 190 & 110 & 50 & 90 & {$[75,85],[100,105]$} \\
\hline
\end{tabular}

Table 8. Transmission loss coefficients for Case IV.

\begin{tabular}{ccccccc}
\hline $\mathbf{B}=\mathbf{1} \times \mathbf{1 0}^{-\mathbf{2}}$ & $\mathbf{0 . 0 0 1 7}$ & $\mathbf{0 . 0 0 1 2}$ & $\mathbf{0 . 0 0 0 7}$ & $\mathbf{- 0 . 0 0 0 1}$ & $\mathbf{- 0 . 0 0 0 5}$ & $\mathbf{- 0 . 0 0 0 2}$ \\
\hline & 0.0012 & 0.0014 & 0.0009 & 0.0001 & -0.0006 & -0.0001 \\
& 0.0007 & 0.0009 & 0.0031 & 0 & -0.001 & -0.0006 \\
& -0.0001 & 0.0001 & 0 & 0.0024 & -0.0006 & -0.0008 \\
& -0.0005 & -0.0006 & -0.001 & -0.0006 & 0.0129 & -0.0002 \\
& -0.0002 & -0.0001 & -0.0006 & -0.0008 & -0.0002 & 0.015 \\
$\mathbf{B}_{\mathbf{0}}=1 \times 10^{-3}$ & -0.3908 & -0.1297 & 0.7047 & 0.0591 & 0.2161 & -0.6635 \\
$\mathbf{B}_{\mathbf{0 0}}=10 \times$ & 0.0056 & & & & & \\
\hline
\end{tabular}

Table 9. Best outputs of different methods for 6-units system.

\begin{tabular}{|c|c|c|c|c|c|c|c|c|c|c|}
\hline Generator & SA [46] & GA [51] & MTS [52] & NPSO [49] & PSO [46] & JAYA [37] & GWO & GWOI & GWOII & NGWO \\
\hline 1 & 478.1258 & 462.0444 & 448.1277 & 447.4734 & 447.5823 & 457.9858 & 446.6281 & 447.2399 & 446.9060 & 448.7973 \\
\hline 2 & 163.0249 & 189.4456 & 172.8082 & 173.1012 & 172.8387 & 176.8785 & 171.7686 & 175.0336 & 172.1000 & 174.4309 \\
\hline 3 & 261.7146 & 254.8535 & 262.5932 & 262.6804 & 261.3300 & 250.0717 & 264.6710 & 262.6065 & 263.8918 & 262.9964 \\
\hline 4 & 125.7665 & 127.4296 & 136.9605 & 139.4156 & 138.6812 & 129.3748 & 141.3356 & 138.8324 & 139.8172 & 138.2484 \\
\hline 5 & 153.7056 & 151.5388 & 168.2031 & 165.3002 & 169.6781 & 172.8886 & 166.5389 & 167.2797 & 164.4018 & 164.9710 \\
\hline 6 & 93.7965 & 90.7150 & 87.3304 & 87.9761 & 85.8963 & 88.4618 & 85.0000 & 85.0000 & 88.8170 & 86.46551 \\
\hline$P_{\text {total }}(\mathrm{MW})$ & 1276.1339 & 1276.0270 & 1276.0232 & 1275.96 & 1276.0066 & 1275.6611 & 1276.3156 & 1276.0229 & 1276.0155 & 1275.4658 \\
\hline$P_{\text {loss }}$ (MW) & 13.1317 & 13.0268 & 13.0205 & 12.9470 & 13.0066 & 12.6665 & 13.3099 & 13.0222 & 13.0066 & 12.8486 \\
\hline$F_{\text {best }}(\$ / \mathrm{h})$ & $15,461.10$ & $15,457.96$ & $15,450.06$ & $15,450.00$ & $15,450.14$ & $15,447.09$ & $15,450.07$ & $15,443.25$ & $15,449.96$ & $15,449.17$ \\
\hline
\end{tabular}

Table 10. Comparison results of 6-units system.

\begin{tabular}{cccc}
\hline Algorithm & $\boldsymbol{F}_{\text {best }} \mathbf{( \$ / h )}$ & $\boldsymbol{F}_{\text {worst }} \mathbf{( \$ / h )}$ & $\boldsymbol{F}_{\text {mean }} \mathbf{( \$ / h )}$ \\
\hline SA [46] & $15,461.10$ & $15,545.50$ & $15,488.98$ \\
GA [51] & $15,457.96$ & $15,524.69$ & $15,477.71$ \\
MTS [52] & $15,450.06$ & $15,453.64$ & $15,451.17$ \\
NPSO [49] & $15,450.00$ & $15,454.00$ & $15,452.00$ \\
PSO [46] & $15,450.14$ & $15,491.71$ & $15,465.83$ \\
JAYA [37] & $15,477.09$ & $15,622.16$ & $15,500.11$ \\
GWO & $15,450.07$ & $15,487.14$ & $15,453.41$ \\
GWOI & $15,450.15$ & $15,455.17$ & $15,451.13$ \\
GWOII & $15,449.96$ & $15,452.41$ & $15,450.48$ \\
NGWO & $15,449.17$ & $15,460.10$ & $15,449.86$ \\
\hline
\end{tabular}




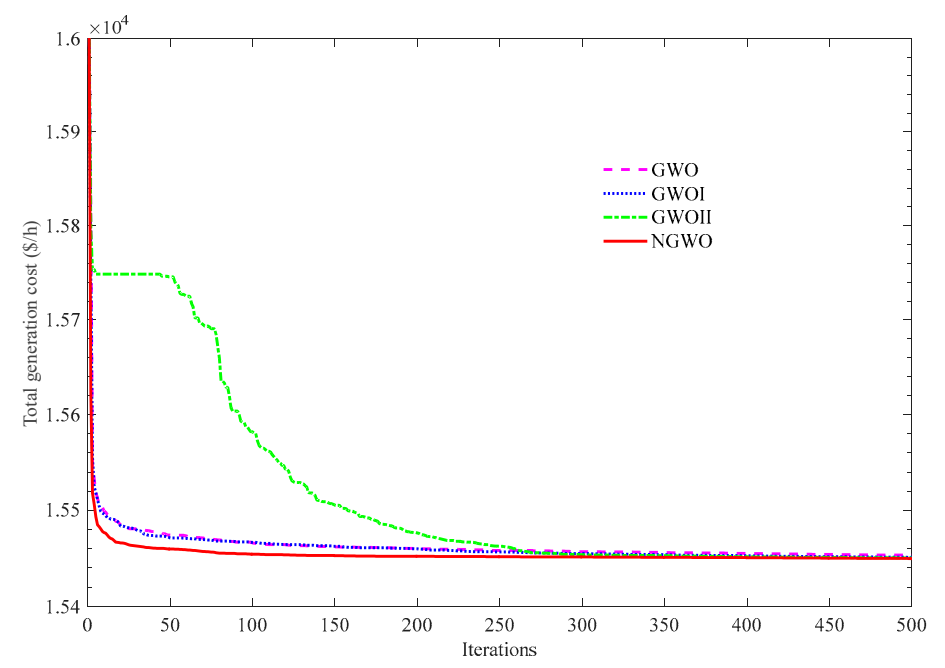

Figure 13. Convergence curve of the total generation cost for the mean solution in Case IV.

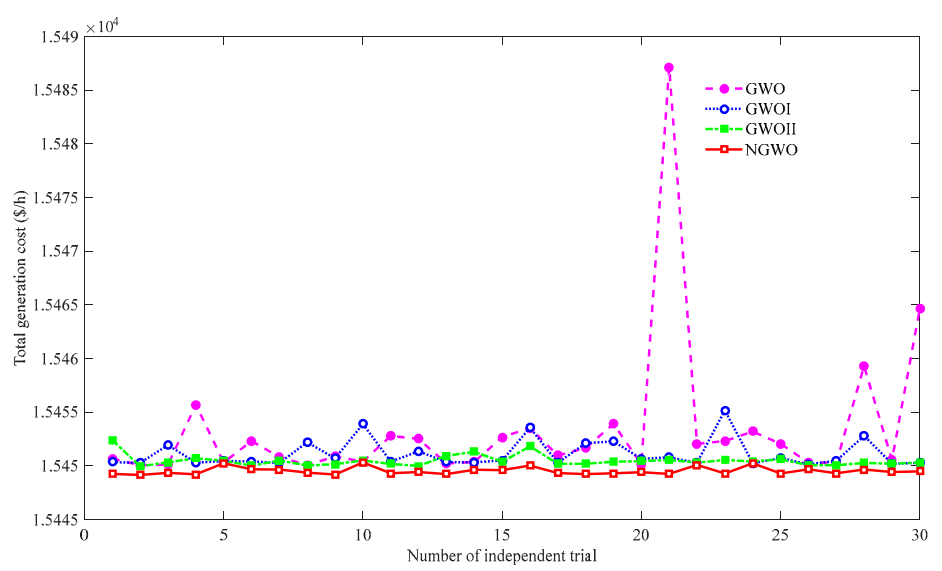

Figure 14. Total generation cost obtained by GWO, GWOI, GWOII, and NGWO for 30 trials in Case IV.

\subsection{Case V: 15-Generator System}

This case study comprises a larger 15-unit system with quadratic cost functions and it has the same constraints as those in case IV. Table 11 shows the generator data and POZs, in which three POZs exist in generators 2, 5, and 6, and generator 12 has two POZs. The transmission loss coefficient data are taken directly from Ref. [53]. The best output results that were achieved by GWO, GWOI, GWOII, and NGWO are compared with those of SA [46], GA [47], MTS [52], TSA [54], PSO [46], and AIS [55], as recorded in Table 12. The table shows that NGWO obtained the lowest best generation cost among all of the abovementioned methods. Table 13 compares the $F_{\text {best }}, F_{\text {worst }}$, and $F_{\text {mean }}$ of the four versions of GWO with those of the techniques that are listed above. As shown in Table 13, NGWO achieved the best $F_{\text {best }}$ and $F_{\text {mean }}$ and the third best $F_{\text {worst }}$. GWOII provided the best $F_{\text {worst, }}$ and MTS obtained the second best $F_{\text {best }}, F_{\text {worst }}$, and $F_{\text {mean }}$. Figure 15 shows the convergence curves of average evaluation values of the 15-generator systems while using the four versions of the GWO method. The NGWO was the fastest algorithm to converge to the global optimal solution as can be seen from the simulation. Figure 16 displays the distribution outline of the best solution in 30 runs and, in most of the trials, the NGWO method obtained a better-quality solution and strong, robust characteristics. 
Table 11. Ramp rate limits and prohibited zones of units for Case V.

\begin{tabular}{cccccccccc}
\hline Generator & $\boldsymbol{P}_{\boldsymbol{i} \text { min }}(\mathbf{M W})$ & $\boldsymbol{P}_{\boldsymbol{i} \max }(\mathbf{M W})$ & $\boldsymbol{a}_{\boldsymbol{i}}$ & $\boldsymbol{b}_{\boldsymbol{i}}$ & $\boldsymbol{c}_{\boldsymbol{i}}$ & $\boldsymbol{P}_{\boldsymbol{i}}^{\mathbf{0}}$ & $\boldsymbol{U} \boldsymbol{R}_{\boldsymbol{i}}$ & $\boldsymbol{D R}_{\boldsymbol{i}}$ & Prohibited Zones \\
\hline 1 & 150 & 455 & 0.000299 & 10.1 & 671 & 400 & 80 & 120 & \\
2 & 150 & 455 & 0.000183 & 10.2 & 574 & 300 & 80 & 120 & {$[185,225],[305,335],[420,450]$} \\
3 & 20 & 130 & 0.001126 & 8.8 & 374 & 105 & 130 & 130 & \\
4 & 20 & 130 & 0.001126 & 8.8 & 374 & 100 & 130 & 130 & \\
5 & 150 & 470 & 0.000205 & 10.4 & 461 & 90 & 80 & 120 & {$[180,200],[305,335],[390,420]$} \\
6 & 135 & 460 & 0.000301 & 10.1 & 630 & 400 & 80 & 120 & {$[230,255],[365,395],[430,455]$} \\
7 & 135 & 465 & 0.000364 & 9.8 & 548 & 350 & 80 & 120 & \\
8 & 60 & 300 & 0.000338 & 11.2 & 227 & 95 & 65 & 100 & \\
9 & 25 & 162 & 0.000807 & 11.2 & 173 & 105 & 60 & 100 & \\
10 & 25 & 160 & 0.001203 & 10.7 & 175 & 110 & 60 & 100 & \\
11 & 20 & 80 & 0.003586 & 10.2 & 186 & 60 & 80 & 80 & \\
12 & 20 & 80 & 0.005513 & 9.9 & 230 & 40 & 80 & 80 & {$[30,40],[55,65]$} \\
13 & 25 & 85 & 0.000371 & 13.1 & 225 & 30 & 80 & 80 & \\
14 & 15 & 55 & 0.001929 & 12.1 & 309 & 20 & 55 & 55 & \\
15 & 15 & 55 & 0.004447 & 12.4 & 323 & 20 & 55 & 55 & \\
\hline
\end{tabular}

Table 12. Best outputs of different methods for 15-units system.

\begin{tabular}{cccccccccccc}
\hline Generator & SA [46] & GA [47] & MTS [53] & TSA [54] & PSO [46] & AIS [55] & SPSO [48] & GWO & GWOI & GWOII & NGWO \\
\hline 1 & 453.6646 & 445.5619 & 453.9922 & 440.500 & 454.7167 & 441.159 & 439.12 & 455.0000 & 455.0000 & 455.0000 & 455.0000 \\
2 & 377.6091 & 380.0000 & 379.7434 & 346.800 & 376.2002 & 409.587 & 407.97 & 380.0000 & 380.0000 & 380.0000 & 380.0000 \\
3 & 120.3744 & 129.0605 & 130.0000 & 110.880 & 129.5547 & 117.298 & 119.63 & 130.0000 & 130.0000 & 130.0000 & 130.0000 \\
4 & 126.2668 & 129.5250 & 129.9232 & 122.460 & 129.7083 & 131.258 & 129.99 & 130.0000 & 130.0000 & 130.0000 & 130.0000 \\
5 & 165.3048 & 169.9659 & 168.0877 & 177.740 & 169.4407 & 151.011 & 151.07 & 170.0000 & 165.3122 & 167.8379 & 160.5430 \\
6 & 459.2455 & 458.7544 & 460.0000 & 459.110 & 458.8153 & 466.258 & 460.00 & 159.0815 & 460.0000 & 460.0000 & 460.0000 \\
7 & 422.8619 & 417.9041 & 429.2253 & 406.410 & 427.5733 & 423.368 & 425.56 & 430.0000 & 430.0000 & 430.0000 & 430.0000 \\
8 & 126.4025 & 97.8230 & 104.3097 & 107.550 & 67.2834 & 99.948 & 98.57 & 102.8806 & 60.8698 & 78.4634 & 84.1915 \\
9 & 54.4742 & 54.2933 & 35.0358 & 107.270 & 75.2673 & 110.684 & 113.49 & 43.5154 & 69.1738 & 48.3551 & 57.7845 \\
10 & 149.0879 & 144.2214 & 155.8829 & 140.560 & 155.5899 & 100.229 & 101.11 & 125.8636 & 158.4501 & 148.3092 & 146.7789 \\
11 & 77.9594 & 77.3302 & 79.8994 & 78.470 & 79.9522 & 32.057 & 33.91 & 80.0000 & 80.0000 & 80.0000 & 80.0000 \\
12 & 93.9489 & 77.0371 & 79.9037 & 74.170 & 79.8947 & 78.815 & 79.96 & 80.0000 & 80.0000 & 80.0000 & 80.0000 \\
13 & 25.0022 & 31.1537 & 25.0220 & 31.950 & 25.2744 & 23.568 & 25.00 & 33.2702 & 30.1938 & 30.5576 & 32.7497 \\
14 & 16.0636 & 15.0233 & 15.2586 & 37.380 & 16.7318 & 40.258 & 41.41 & 26.4876 & 17.6755 & 18.5833 & 17.2977 \\
15 & 15.0196 & 33.6125 & 15.0796 & 22.470 & 15.1967 & 36.906 & 35.61 & 15.0116 & 15.1082 & 23.69718 & 15.4832 \\
$P_{\text {total }}$ (MW) & 2663.29 & 2661.23 & 2661.36 & 2663.70 & 2661.19 & 2662.04 & 2662.4 & 2662.2318 & 2662.1458 & 2660.96 & 2660.54 \\
$P_{\text {loss }}$ (MW) & 33.2737 & 31.2363 & 31.3523 & 33.8110 & 31.1697 & 32.4075 & 32.431 & 32.2317 & 31.0156 & 30.8550 \\
$F_{\text {best }}(\$ / \mathrm{h})$ & $32,786.40$ & $32,779.81$ & $32,716.87$ & $32,918.00$ & $32,724.17$ & $32,854.00$ & 32,858 & $32,743.2959$ & $32,733.8961$ & $32,734.6249$ & 30.0148 \\
\hline
\end{tabular}

Table 13. Comparison results of 15-units system.

\begin{tabular}{cccc}
\hline Algorithm & $\boldsymbol{F}_{\text {best }} \mathbf{( \$ / h )}$ & $\boldsymbol{F}_{\text {worst }} \mathbf{( \$ / h )}$ & $\boldsymbol{F}_{\text {mean }} \mathbf{( \$ / h )}$ \\
\hline SA [46] & $32,786.40$ & $33,028.95$ & $32,869.51$ \\
GA [47] & $32,779.81$ & $33,041.64$ & $32,841.21$ \\
MTS [53] & $32,716.87$ & $32,796.15$ & $32,767.21$ \\
TSA [54] & $32,917.87$ & $33,245.54$ & $33,066.76$ \\
PSO [46] & $32,724.17$ & $32,841.38$ & $32,807.45$ \\
SPSO [48] & $32,858.00$ & $33,331.00$ & $33,039.00$ \\
AIS [55] & $32,854.00$ & $32,892.00$ & $32,873.25$ \\
GWO & $32,743.30$ & $32,857.96$ & $32,784.96$ \\
GWOI & $32,733.90$ & $32,889.63$ & $32,783.22$ \\
GWOII & $32,734.62$ & $32,817.91$ & $32,774.82$ \\
NGWO & $32,712.61$ & $32,830.61$ & $32,752.78$ \\
\hline
\end{tabular}




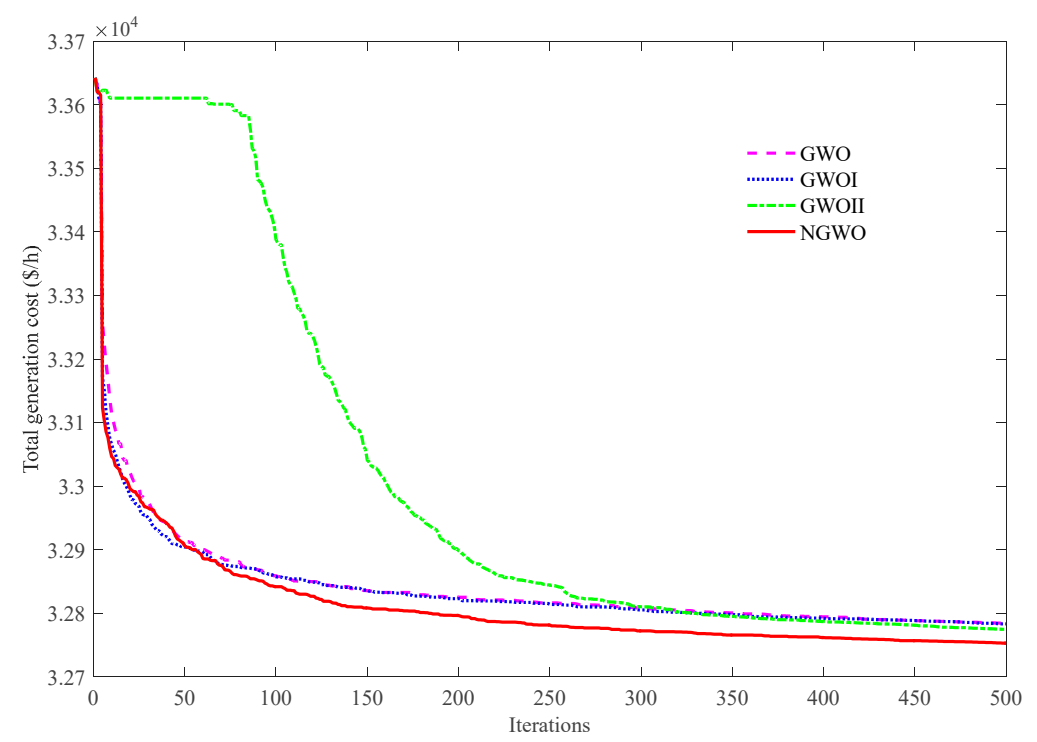

Figure 15. Convergence curve of the total generation cost for the mean solution in Case V.

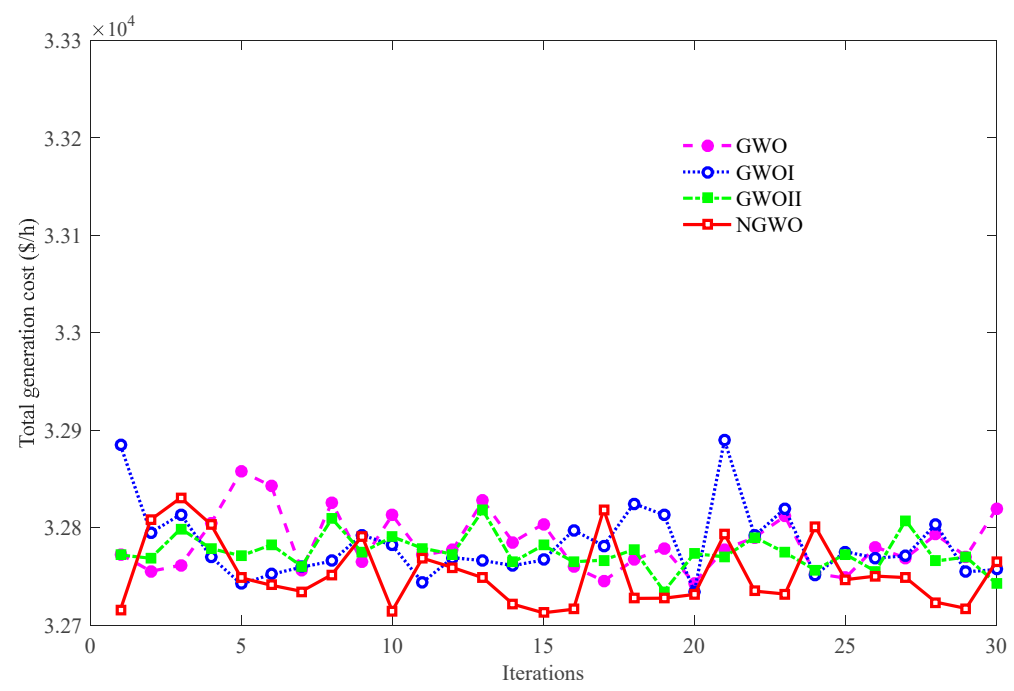

Figure 16. Total generation cost obtained by GWO, GWOI, GWOII, and NGWO for 30 trials in Case V.

\section{Conclusions and Future Work}

In this paper, we successfully applied the proposed NGWO technique to solve the ELD problem by considering the ramp rate limits, POZ constraints, and nonsmooth cost functions. The NGWO algorithm was validated to improve both the global exploration capability and the convergence rates and it had the best robustness when compared to the other three versions of the GWO algorithms. Furthermore, the results, when compared to all the other compared algorithms in five cases, demonstrated the outstanding superiority of the NGWO method in solving the ELD problem.

The superiority of the NGWO algorithm in solving ELD problems was proven. Although our research has not been yet applied to any utility companies or energy providers, we believe that the application of this algorithm in the future will surely improve the operational level of these companies. Next, an interesting application would be to apply the algorithm to training neural networks, optimizing restrictive engineering structures, and solving multiobjective optimization problems. However, for the large-scale power systems of Cases II, III, and V, the robustness of the NGWO algorithm in solving these problems is not as perfect as in solving Cases I and IV, so the optimization performance of the NGWO algorithm can still be improved. 
Author Contributions: Conceptualization, F.Y. and J.X.; methodology, F.Y.; software, F.Y.; validation, F.Y., K.Y. and F.L.; formal analysis, F.Y.; investigation, F.Y.; resources, F.Y.; data curation, J.G.; writing-original draft preparation, F.Y.; writing—review and editing, F.Y.; visualization, L.S.; supervision, J.X.; project administration, J.X.; funding acquisition, J.X.

Funding: This research was funded by the National Natural Science Foundation of China, grant number 71841054, the Philosophy and Social Science Research Plan of Heilongjiang Province, grant number 18JYC257, the Young Innovative Talents Training Program for Universities in Heilongjiang Province, grant number UNPYSCT-2018151, and the Fundamental Research Funds for the Central Universities, grant number 2572018BM07.

Acknowledgments: We are grateful to the anonymous reviewers for their valuable comments that helped us improve this paper.

Conflicts of Interest: The authors declare no conflict of interest.

\section{References}

1. Niknam, T.; Mojarrad, H.D.; Meymand, H.Z.; Firouzi, B.B. A new honey bee mating optimization algorithm for non-smooth economic dispatch. Energy 2011, 36, 896-908. [CrossRef]

2. Narimani, M.R.; Joo, J.-Y.; Crow, M.L. Dynamic Economic Dispatch with Demand Side Management of Individual Residential Loads. In Proceedings of the 2015 North American Power Symposium (NAPS), Charlotte, NC, USA, 4-6 October 2015; pp. 1-6.

3. Chen, C.L.; Wang, S.C. Branch-and-bound scheduling for thermal generating units. IEEE Trans. Energy Convers. 1993, 8, 184-189. [CrossRef]

4. Papageorgiou, L.G.; Fraga, E.S. A mixed integer quadratic programming formulation for the economic dispatch of generators with prohibited operating zones. Electr. Power Syst. Res. 2007, 77, 1292-1296. [CrossRef]

5. Dodu, J.C.; Martin, P.; Merlin, A.; Pouget, J. An optimal formulation and solution of short-range operating problems for a power system with flow constraints. Proc. IEEE 1972, 60, 54-63. [CrossRef]

6. Irisarri, G.; Kimball, L.M.; Clements, K.A.; Bagchi, A.; Davis, P.W. Economic dispatch with network and ramping constraints via interior point methods. IEEE Trans. Power Syst. 1998, 13, 236-242. [CrossRef]

7. Farag, A.; Al-Baiyat, S.; Cheng, T.C. Economic load dispatch multiobjective optimization procedures using linear programming techniques. IEEE Trans. Power Syst. 1995, 10, 731-738. [CrossRef]

8. Liang, Z.X.; Glover, J.D. A zoom feature for a dynamic programming solution to economic dispatch including transmission losses. IEEE Trans. Power Syst. 1992, 7, 544-550. [CrossRef]

9. Li, Z.; Wu, W.; Zhang, B.; Sun, H.; Guo, Q. Dynamic economic dispatch using Lagrangian relaxation with multiplier updates based on a quasi-Newton method. IEEE Trans. Power Syst. 2013, 28, 4516-4527. [CrossRef]

10. Niknam, T. A new fuzzy adaptive hybrid particle swarm optimization algorithm for non-linear, non-smooth and non-convex economic dispatch problem. Appl. Energy 2010, 87, 327-339. [CrossRef]

11. Modiri-Delshad, M.; Kaboli, S.H.A.; Taslimi-Renani, E.; Abd Rahim, N. Backtracking search algorithm for solving economic dispatch problems with valve-point effects and multiple fuel options. Energy 2016, 116, 637-649. [CrossRef]

12. Chiang, C.L. Improved genetic algorithm for power economic dispatch of units with valve-point effects and multiple fuels. IEEE Trans. Power Syst. 2005, 20, 1690-1699. [CrossRef]

13. Park, J.B.; Lee, K.S.; Shin, J.R.; Lee, K.S. A particle swarm optimization for economic dispatch with nonsmooth cost functions. IEEE Trans. Power Syst. 2005, 20, 34-42. [CrossRef]

14. Basu, M.; Chowdhury, A. Cuckoo search algorithm for economic dispatch. Energy 2013, 60, 99-108. [CrossRef]

15. Secui, D.C. A new modified artificial bee colony algorithm for the economic dispatch problem. Energy Convers. Manag. 2015, 89, 43-62. [CrossRef]

16. Adarsh, B.R.; Raghunathan, T.; Jayabarathi, T.; Yang, X.-S. Economic dispatch using chaotic bat algorithm. Energy 2016, 96, 666-675. [CrossRef]

17. Coelho, L.D.S.; Mariani, V.C. An improved harmony search algorithm for power economic load dispatch. Energy Convers. Manag. 2009, 50, 2522-2526. [CrossRef]

18. Pradhan, M.; Roy, P.K.; Pal, T. Grey wolf optimization applied to economic load dispatch problems. Int. J. Electr. Power Energy Syst. 2016, 83, 325-334. [CrossRef]

19. Jayabarathi, T.; Raghunathan, T.; Adarsh, B.R.; Suganthan, P.N. Economic dispatch using hybrid grey wolf optimizer. Energy 2016, 111, 630-641. [CrossRef] 
20. Niknam, T.; Mojarrad, H.D.; Meymand, H.Z. A novel hybrid particle swarm optimization for economic dispatch with valve-point loading effects. Energy Convers. Manag. 2011, 52, 1800-1809. [CrossRef]

21. Wang, L.; Li, L.P. An effective differential harmony search algorithm for the solving non-convex economic load dispatch problems. Int. J. Electr. Power Energy Syst. 2013, 44, 832-843. [CrossRef]

22. Park, J.-B.; Jeong, Y.-W.; Shin, J.-R.; Lee, K.Y. An improved particle swarm optimization for nonconvex economic dispatch problems. IEEE Trans. Power Syst. 2010, 25, 156-166. [CrossRef]

23. Wolpert, D.H.; Macready, W.G. No free lunch theorems for optimization. IEEE Trans. Evol. Comput. 1997, 1, 67-82. [CrossRef]

24. Mirjalili, S.; Mirjalili, S.M.; Lewis, A. Grey Wolf Optimizer. Adv. Eng. Softw. 2014, 69, 46-61. [CrossRef]

25. Xu, J.; Yan, F. Hybrid Nelder-Mead Algorithm and Dragonfly Algorithm for Function Optimization and the Training of a Multilayer Perceptron. Arab. J. Sci. Eng. 2019, 44, 3473-3487. [CrossRef]

26. Xu, J.; Yan, F.; Yun, K.; Ronald, S.; Li, F.; Guan, J. Dynamically Dimensioned Search Embedded with Piecewise Opposition-Based Learning for Global Optimization. Sci. Program. 2019, 2019, 2401818. [CrossRef]

27. Faris, H.; Aljarah, I.; Al-Betar, M.A.; Mirjalili, S. Grey wolf optimizer: A review of recent variants and applications. Neural Comput. Appl. 2018, 30, 413-435. [CrossRef]

28. Emary, E.; Zawbaa, H.M.; Hassanien, A.E. Binary grey wolf optimization approaches for feature selection. Neurocomputing 2016, 172, 371-381. [CrossRef]

29. Mirjalili, S. How effective is the grey wolf optimizer in training multi-layer perceptrons? Appl. Intell. 2015, 43, 150-161. [CrossRef]

30. Mustaffa, Z.; Sulaiman, M.H.; Kahar, M.N.M. Training lssvm with gwo for price forecasting. In Proceedings of the 2015 International Conference on Informatics, Electronics \& Vision (ICIEV), Kitakyushu, Fukuoka, Japan, 15-18 June 2015; pp. 1-6.

31. Zhang, S.; Zhou, Y. Grey wolf optimizer based on Powell local optimization method for clustering analysis. Discret Dyn. Nat. Soc. 2015, 2015, 481360. [CrossRef]

32. Li, S.X.; Wang, J.S. Dynamic modeling of steam condenser and design of pi controller based on grey wolf optimizer. Math. Probl. Eng. 2015, 2015, 120975. [CrossRef]

33. Song, H.M.; Sulaiman, M.H.; Mohamed, M.R. An application of grey wolf optimizer for solving combined economic emission dispatch problems. Int. Rev. Model Simul. 2014, 7, 838-844.

34. Zhang, S.; Zhou, Y.; Li, Z.; Pan, W. Grey wolf optimizer for unmanned combat aerial vehicle path planning. Adv. Eng. Softw. 2016, 99, 121-136. [CrossRef]

35. Lu, C.; Xiao, S.; Li, X.; Gao, L. An effective multi-objective discrete grey wolf optimizer for a real-world scheduling problem in welding production. Adv. Eng. Softw. 2016, 99, 161-176. [CrossRef]

36. Sheng, W.G.; Chen, S.Y.; Sheng, M.M.; Mao, J.F.; Zheng, Y.J. Adaptive Multisubpopulation Competition and Multiniche Crowding-Based Memetic Algorithm for Automatic Data Clustering. IEEE Trans. Evol. Comput. 2016, 20, 838-858.

37. Wang, Y.-K.; Chen, X.-B. Improved multi-area search and asymptotic convergence PSO algorithm with independent local search mechanism. Control. Decis. 2018, 33, 1382-1389.

38. Al-Betar, M.A.; Awadallah, M.A.; Khader, A.T.; Bolaji, A.L.; Almoman, A. Economic load dispatch problems with valve-point loading using natural updated harmony search. Neural Comput. Appl. 2018, 29, 767-781. [CrossRef]

39. Yu, J.; Kim, C.-H.; Abdul, W.; Tahir, K.; Rhee, S.-B. A Novel Multi-Population Based Chaotic JAYA Algorithm with Application in Solving Economic Load Dispatch Problems. Energies 2018, 11, 1946. [CrossRef]

40. Vrkalovic, S.; Lunca, E.-C.; Borlea, I.-D. Model-Free Sliding Mode and Fuzzy Controllers for Reverse Osmosis Desalination Plants. Int. J. Artif. Intell. 2018, 16, 208-222.

41. Shams, M.; Rashedi, E.; Dashti, S.M.; Hakimi, A. Ideal Gas Optimization Algorithm. Int. J. Artif. Intell. 2017, 15, 116-130.

42. Vašcák, J. Adaptation of fuzzy cognitive maps by migration algorithms. Kybernetes 2012, 41, 429-443. [CrossRef]

43. Precup, R.-E.; David, R.-C. Nature-inspired Optimization Algorithms for Fuzzy Controlled Servo Systems; Butterworth-Heinemann, Elsevier: Oxford, UK, 2019; pp. 1-48.

44. Coelho, L.S.; Lee, C.-S. Solving economic load dispatch problems in power systems using chaotic and Gaussian particle swarm optimization approaches. Int. J. Elec. Power. 2008, 30, 297-307. [CrossRef] 
45. Walters, D.C.; Sheble, G.B. Genetic algorithm solution of economic dispatch with valve point loading. IEEE T. Power Syst. 1993, 8, 1325-1332. [CrossRef]

46. Victoire, T.A.A.; Jeyakumar, A.E. Hybrid PSO-SQP for economic dispatch with valve-point effect. Electr. Power Syst. Res. 2004, 71, 51-59. [CrossRef]

47. Sinha, N.; Chakrabarti, R.; Chattopadhyay, P.K. Evolutionary programming techniques for economic load dispatch. IEEE Trans. Evol. Comput. 2003, 7, 83-94. [CrossRef]

48. Cai, J.; Li, Q. A hybrid CPSO-SQP method for economic dispatch considering the valve-point effects. Energy Convers. Manag. 2012, 53, 175-181. [CrossRef]

49. Selvakumar, I.; Thanushkodi, K. A new particle swarm optimization solution to nonconvex economic dispatch problems. Electr. Power Syst. Res. 2007, 22, 42-51. [CrossRef]

50. Ling, S.H.; Leung, F.H.F. An Improved genetic algorithm with average-bound crossover and wavelet mutation operation. Soft Comput. 2007, 11, 7-31. [CrossRef]

51. Bhagwan Das, D.; Patvardhan, C. Solution of Economic Load Dispatch using real coded Hybrid Stochastic Search. Int. J. Electr. Power Energy Syst. 1999, 21, 165-170. [CrossRef]

52. Pothiya, S.; Ngamroo, I.; Kongprawechnon, W. Application of multiple tabu search algorithm to solve dynamic economic dispatch considering generator constraints. Energy Convers Manag. 2008, 49, 506-516. [CrossRef]

53. Gaing, Z.-L. Particle swarm optimization to solving the economic dispatch considering the generator constraints. IEEE Trans. Power Syst. 2003, 18, 1187-1195. [CrossRef]

54. Khamsawang, S.; Jiriwibhakorn, S. DSPSO-TSA for economic dispatch problem with nonsmooth and noncontinuous cost functions. Energy Convers Manag. 2010, 51, 365-375. [CrossRef]

55. Panigrahi, B.K.; Yadav, S.R.; Agrawal, S.; Tiwari, M.K. A clonal algorithm to solve economic load dispatch. Electr. Power Syst. Res. 2007, 77, 1381-1389. [CrossRef]

(C) 2019 by the authors. Licensee MDPI, Basel, Switzerland. This article is an open access article distributed under the terms and conditions of the Creative Commons Attribution (CC BY) license (http://creativecommons.org/licenses/by/4.0/). 